\title{
Synthesis of Some 4,5-Dihydrothieno[3,2-e][1,2,4]Triazolo[4,3-a] Pyrimi- dine-2-Carboxamides as Anti-Inflammatory and Analgesic Agents
}

\author{
Omaima G. Shaaban ${ }^{1}$, Ola H. Rizk ${ }^{1, *}$, Aida E. Bayad ${ }^{2}$ and Ibrahim M.El-Ashmawy ${ }^{3}$ \\ ${ }^{1}$ Department of Pharmaceutical Chemistry, Faculty of Pharmacy, University of Alexandria, Alexandria 21521, Egypt; \\ ${ }^{2}$ Univ. Fellow, Veterinary Services Unit, Faculty of Veterinary Medicine, University of Alexandria, Egypt; ${ }^{3}$ Department \\ of Veterinary Medicine, Faculty of Agricultural and Veterinary Medicine, Qassim University, Buraydah 1482, Al- \\ Qassim, Saudi Arabia
}

\begin{abstract}
A new series 4,5-dihydrothieno[3,2-e][1,2,4]triazolo[4,3-a]pyrimidine-2-carboxamide was synthesized. Twenty one newly synthesized compounds were investigated for their anti-inflammatory and analgesic activity using acute and subacute formalin-induced paw edema models and diclofenac $\mathrm{Na}$ as a reference. The acute toxicity $\left(\mathrm{ALD}_{50}\right)$ and ulcerogenic effects of the active compounds were also determined. The thienotriazolopyrimidines 10a, 10c and 11c were found to exhibit remarkable anti-inflammatory activity at both models in addition to good analgesic activity with a delayed onset of action. Moreover, the active compounds showed high GI safety level and are well tolerated by experimental animals with high safety margin $\left(\mathrm{ALD}_{50}>0.4 \mathrm{~g} / \mathrm{kg}\right)$. Docking study using Molecular Operating Environment (MOE) version 2008.10 into COX-2 has been made for derivatives of highest anti-inflammatory activity.
\end{abstract}

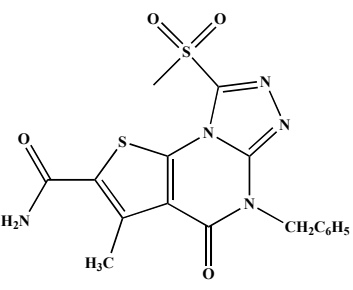

10a

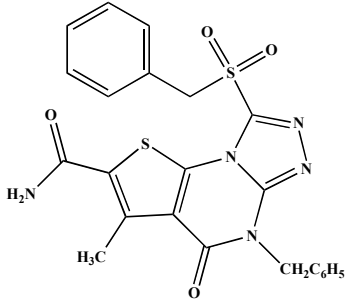

$10 \mathrm{c}$

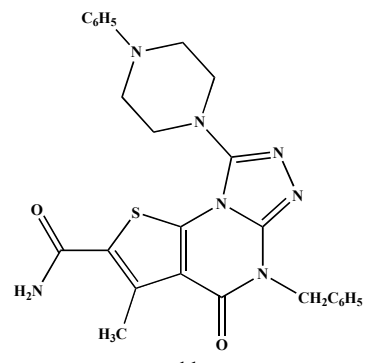

$11 \mathrm{c}$

Thienotriazolopyrimidine derivatives with promising anti-inflammatory and analgesic activities.

Keywords: Synthesis, thienotriazolopyrimidines, antiinflammatory activity, ulcerogenic effect, acute toxicity, analgesic activity, docking.

\section{INTRODUCTION}

It is well known that prolonged use of non steroidal antiinflammatory drugs (NSAIDs) is associated with several side effects such as gastrointestinal mucosal damage, bleeding, intolerance and renal toxicity $[1,2]$. Therefore, production of safer and more active NSAIDs and analgesic drugs is still needed.

It is evident from the literature that thienopyrimidines, played an essential role in several biological processes and have considerable chemical and pharmacological importance. They were found to display antiviral $[3,4]$, antimicrobial [5-8], anticancer [5, 9-11], analgesic and antiinflammatory activities [12-16].

*Address correspondence to this author at the Department of Pharmaceutical Chemistry, Faculty of Pharmacy, University of Alexandria, Alexandria 21521, Egypt; Tel: +(203)4871317 ; Fax: + (203)4873273;

Email: olarizk@yahoo.com
In our ongoing medicinal chemistry research programme, we have demonstrated that some dihydrothieno[2,3-d] pyrimidine and tetrahydrothieno[3,2-e][1,2,4]triazolo[4,3a]pyrimidine derivatives (Fig. 1, A-C)[17], exhibited antiinflammatory activity equivalent to diclofenac sodium in the inflammatory models in addition to their high safety level and low ulcerogenicity. Whereas, 2-substituted-6,7-dimethylthieno[2,3- $d][1,2,4]$ triazolo $[1,5-a]$ pyrimidinones and dihydrothieno[2,3-d][1,2,4] triazolo[1,5-a]pyrimidin-2-yl) acetamide (Fig. 2, D and E) [18] exhibited good analgesic and anti-inflammatory activities.

Based on these findings we extended our approach to the synthesis and pharmacological investigation of some new dihydrothieno[3,2-e][1,2,4] triazolo[4,3-a]pyrimidine-2carboxamides as anti-inflammatory and analgesic agents. To explore the biological profile of the newly synthesized compounds, the substitution pattern at position 8 of the 

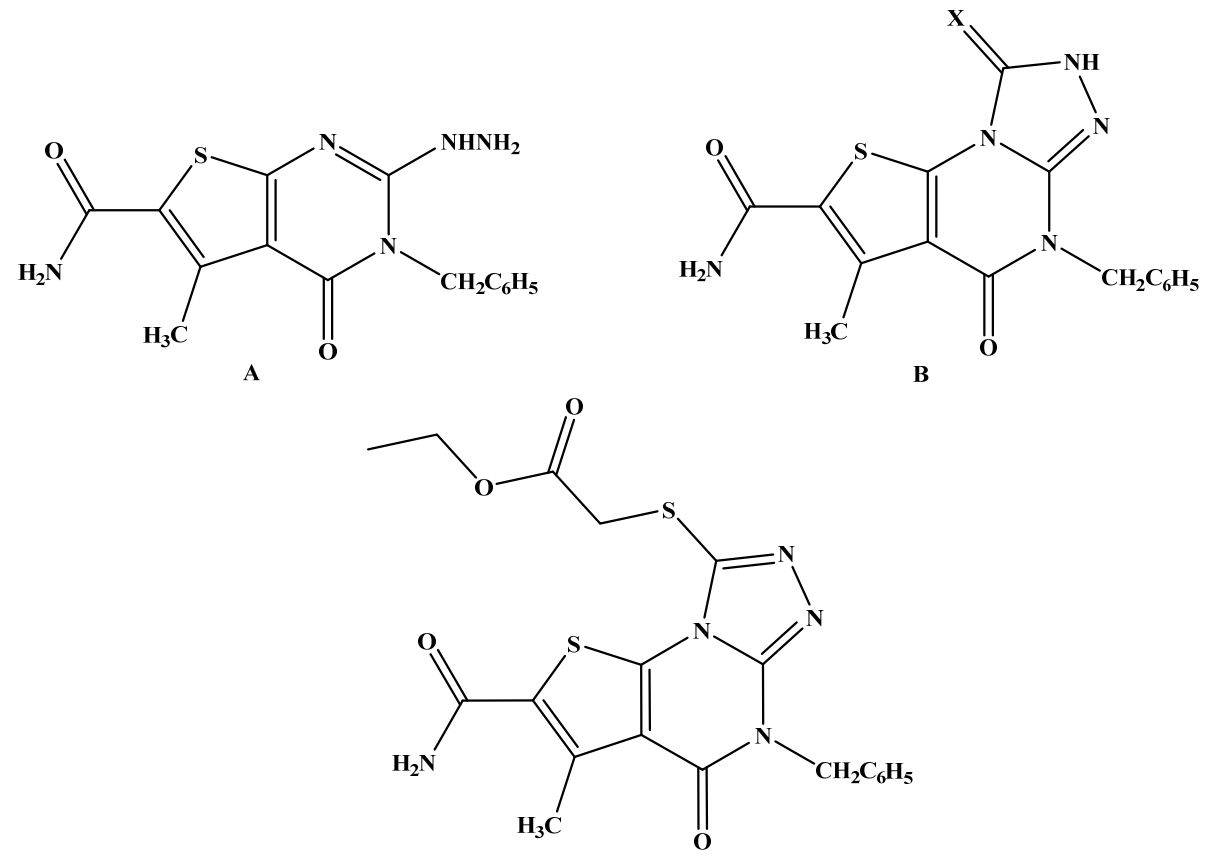

C

Fig. (1). Chemical structures of previously reported anti-inflammatory thienopyrimidines and fused thienopyrimidines.

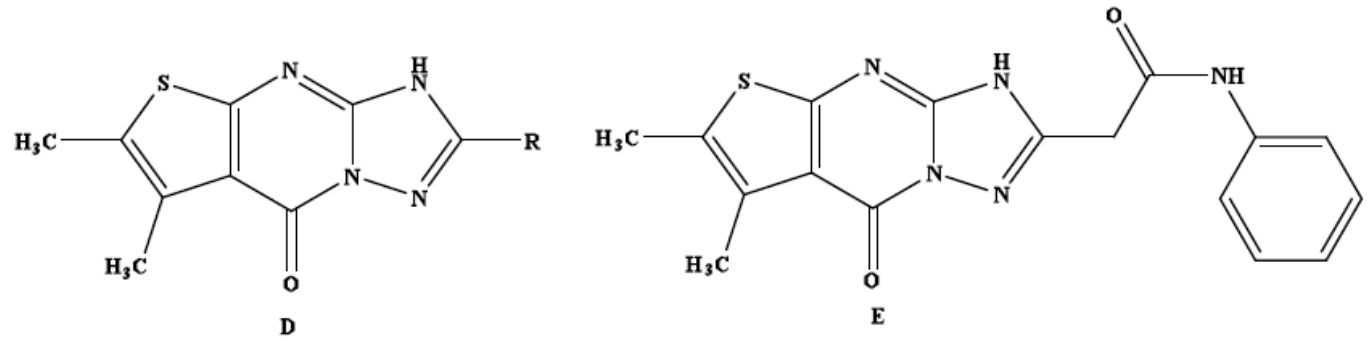

Fig. (2). Chemical structures of previously reported anti-inflammatory and analgesic thienotriazolopyrimidines.

thienotriazolopyrimidine ring system was selected so as to confer different electronic environment that would affect the lipophilicity, and hence the activity of the target molecules. In addition to the anti-inflammatory and analgesic activities, the ulcerogenicity and acute toxicity of the most active compounds were also investigated. A molecular docking study was also performed for the most active compounds in a trial to investigate the possible mode of action of these compounds.

\section{MATERIALS AND METHODS}

\section{Chemistry}

All reagents and solvents were purchased from commercial suppliers and were dried and purified when necessary by standard techniques.

Melting points were determined in open glass capillaries using Stuart capillary melting point apparatus (Stuart Scientific Stone, Staffordshire, UK) and are uncorrected. Infrared (IR) spectra were recorded on Perkin-Elmer 1430 infrared spectrophotometer (Perkin Elmer, Beaconsfield, UK) and measured by v́ $\mathrm{cm}^{-1}$ scale using $\mathrm{KBr}$ cell. ${ }^{1} \mathrm{H}-\mathrm{NMR}$ spectra were scanned on Varian Mercury VX-300, using tetramethylsilane (TMS) as internal standard and DMSO- $\mathrm{d}_{6}$ as the solvent (chemical shifts are given in $\delta \mathrm{ppm}$ ). Splitting patterns were designated as follows: s: singlet; d: doublet; $\mathrm{dd}$ : doublet of doublet; $\mathrm{t}$ : triplet; $\mathrm{m}$ : multiplet. ${ }^{13} \mathrm{C}-\mathrm{NMR}$ proton decoupled spectra were recorded on a Varian Mercury VX-300 spectrometer in DMSO- $\mathrm{d}_{6}$ and measured in $\delta$ scale. Mass spectra were run on a Finnigan mass spectrometer model SSQ/7000 (70 eV) or on a gas chromatograph/mass spectrometer Schimadzu GCMS-QP 2010 Plus (70 eV). Microanalyses were performed at the Microanalytical Unit, Faculty of Science, Cairo University, Egypt and the found values were within $\pm 0.4 \%$ of the theoretical values. Follow up of the reactions and checking the purity of the compounds was made by thin layer chromatography (TLC) on silica gelprecoated aluminium sheets (Type 60 GF254; Merck; Germany) and the spots were detected by exposure to UV lamp at $\lambda 254 \mathrm{~nm}$ for few seconds. The key intermediate 5 was synthesized as described in Ref. [17] 
5- Benzyl-3-methyl-8-\{[2-(substituted amino)-2oxoethyl]sulfanyl\}-4-oxo-4,5-dihydrothieno[3,2-e] $[1,2,4]$ triazolo[4,3-a]pyrimidine-2-carboxamides (7a-f)

A mixture of 5 ( $0.37 \mathrm{~g}, 1 \mathrm{mmol})$, appropriate 2-chloro$\mathrm{N}$-substituted acetamides 6 ( $1 \mathrm{mmol})$ and anhydrous potassium carbonate $(0.14 \mathrm{~g}, 1 \mathrm{mmol})$ in dry acetone $(20 \mathrm{ml})$ was stirred and heated under reflux for 10 hours. The mixture was then cooled, poured into ice-cold water, filtered and the solid obtained was dried and crystallized from DMF.

5- Benzyl-3-methyl-8-\{[2-(phenylamino)-2oxoethyl]sulfanyl $\}-4-0 \times 0-4,5-d i h y d r o t h i e n o[3,2-e]$ $[1,2,4]$ triazolo $[4,3-a]$ pyrimidine-2-carboxamide (7a)

M.P. 276-8 ${ }^{\circ} \mathrm{C}$; Yield 70\%; IR (KBr, $\left.\mathrm{cm}^{-1}\right): 3395,3345$ $\left(\mathrm{NH}_{2}\right), 3277(\mathrm{NH}), 1679,1658(\mathrm{C}=\mathrm{O}), 1589(\mathrm{C}=\mathrm{N}), 1265$, 1083 (C-S-C). ${ }^{1} \mathrm{H}-\mathrm{NMR}(\delta \mathrm{ppm}): 2.68\left(\mathrm{~s}, 3 \mathrm{H}, \mathrm{CH}_{3}\right), 3.89$ (s, $\left.2 \mathrm{H}, \mathrm{SCH}_{2}\right), 5.28\left(\mathrm{~s}, 2 \mathrm{H}, \mathrm{N}-\mathrm{CH}_{2}\right), 6.98-7.37(\mathrm{~m}, 10 \mathrm{H}$, two phenyl-H), 7.75 (s, 2H, $\mathrm{NH}_{2}, \mathrm{D}_{2} \mathrm{O}$ exchangeable), 9.98 (s, $1 \mathrm{H}, \mathrm{NH}, \mathrm{D}_{2} \mathrm{O}$ exchangeable). Anal. Calcd. for $\mathrm{C}_{24} \mathrm{H}_{20} \mathrm{~N}_{6} \mathrm{O}_{3} \mathrm{~S}_{2}$ (504.58): C, 57.13; H, 4.00; N, 16.66. Found: C, 56.89; H, 3.76; N, 16.34 .

\section{5- Benzyl-3-methyl-8-\{[2-(4-chlorophenylamino)-2- oxoethyl]sulfanyl $\}-4-0 x 0-4,5-d i h y d r o t h i e n o[3,2-e]$ $[1,2,4]$ triazolo[4,3-a]pyrimidine-2-carboxamide (7b)}

M.P. 244-6 ${ }^{\circ} \mathrm{C}$; Yield 68\%; IR $\left(\mathrm{KBr}, \mathrm{cm}^{-1}\right): 3491,3365$ $\left(\mathrm{NH}_{2}\right), 3297(\mathrm{NH}), 1689,1650(\mathrm{C}=\mathrm{O}), 1587(\mathrm{C}=\mathrm{N}), 1267$, 1092 (C-S-C). ${ }^{1} \mathrm{H}-\mathrm{NMR}(\delta \mathrm{ppm}): 2.71\left(\mathrm{~s}, 3 \mathrm{H}, \mathrm{CH}_{3}\right), 3.91(\mathrm{~s}$, $\left.2 \mathrm{H}, \mathrm{SCH}_{2}\right), 5.29\left(\mathrm{~s}, 2 \mathrm{H}, \mathrm{N}-\mathrm{CH}_{2}\right), 7.25-7.43$ (m, 9H, phenyl-H and 4-chlorophenyl-H), $7.79\left(\mathrm{~s}, 2 \mathrm{H}, \mathrm{NH}_{2}, \mathrm{D}_{2} \mathrm{O}\right.$ exchangeable), 10.30 (s, 1H, NH, $\mathrm{D}_{2} \mathrm{O}$ exchangeable). Anal. Calcd. for $\mathrm{C}_{24} \mathrm{H}_{19} \mathrm{ClN}_{6} \mathrm{O}_{3} \mathrm{~S}_{2}$ (539.03): C, 53.48; $\mathrm{H}, 3.55 ; \mathrm{N}, 15.59$. Found: C, 53.19; H, 3.76; N, 15.24.

\section{5- Benzyl-3-methyl-8-\{[2-(4-methylphenylamino)-2- oxoethyl] sulfanyl $\}-4-0 x 0-4,5-d i h y d r o t h i e n o ~[3,2-e]$ $[1,2,4]$ triazolo[4,3-a]pyrimidine-2-carboxamide (7c)}

M.P. 288-90 ${ }^{\circ} \mathrm{C}$; Yield 69\%; IR $\left(\mathrm{KBr}, \mathrm{cm}^{-1}\right): 3382,3364$ $\left(\mathrm{NH}_{2}\right), 1686,1644(\mathrm{C}=\mathrm{O}), 1598(\mathrm{C}=\mathrm{N}), 1252,1062(\mathrm{C}-\mathrm{S}-\mathrm{C})$. ${ }^{1} \mathrm{H}-\mathrm{NMR}(\delta \mathrm{ppm}): 2.23\left(\mathrm{~s}, 3 \mathrm{H}, 4-\mathrm{CH}_{3} \mathrm{C}_{6} \mathrm{H}_{4}\right), 2.74(\mathrm{~s}, 3 \mathrm{H}$, $\left.\mathrm{CH}_{3}\right), 3.93\left(\mathrm{~s}, 2 \mathrm{H}, \mathrm{S}-\mathrm{CH}_{2}\right), 5.33\left(\mathrm{~s}, 2 \mathrm{H}, \mathrm{N}-\mathrm{CH}_{2}\right), 7.06(\mathrm{~d}, \mathrm{~J}=$ $6.9 \mathrm{~Hz}, 2 \mathrm{H}$, 4-methylphenyl- $\left.\mathrm{C}_{3}, 5-\mathrm{H}\right), 7.28-7.35(\mathrm{~m}, 5 \mathrm{H}$, phenyl-H), 7.42 (d, J =6.9 Hz, 2H, 4-methylphenyl- $\left.\mathrm{C}_{2,6}-\mathrm{H}\right)$, 7.79 (s, 2H, $\mathrm{NH}_{2}, \mathrm{D}_{2} \mathrm{O}$ exchangeable), $9.94\left(\mathrm{~s}, 1 \mathrm{H}, \mathrm{NH}, \mathrm{D}_{2} \mathrm{O}\right.$ exchangeable). Anal. Calcd. for $\mathrm{C}_{25} \mathrm{H}_{22} \mathrm{~N}_{6} \mathrm{O}_{3} \mathrm{~S}_{2}$ (518.61): C, 57.90; H, 4.28; N, 16.20. Found: C, 57.98; H, 4.35; N, 16.32.

\section{5- Benzyl-3-methyl-8-\{[2-(4-methoxyphenylamino)-2-} oxoethyl] sulfanyl\}-4-oxo-4,5-dihydrothieno $[3,2-e]$ $[1,2,4]$ triazolo[4,3-a]pyrimidine-2-carboxamide (7d)

M.P. 255-7 ${ }^{\circ} \mathrm{C}$; Yield 69\%; IR $\left(\mathrm{KBr}, \mathrm{cm}^{-1}\right): 3375,3298$ $\left(\mathrm{NH}_{2}\right), 3266(\mathrm{NH}), 1684,1651(\mathrm{C}=\mathrm{O}), 1597,1552(\mathrm{C}=\mathrm{N})$, 1247, 1073 (C-S-C). ${ }^{1} \mathrm{H}-\mathrm{NMR}(\delta \mathrm{ppm}): 2.85$ (s, 3H, $\left.\mathrm{CH}_{3}\right)$, $3.67\left(\mathrm{~s}, 3 \mathrm{H}, \mathrm{OCH}_{3}\right), 3.87\left(\mathrm{~s}, 2 \mathrm{H}, \mathrm{S}-\mathrm{CH}_{2}\right), 5.29(\mathrm{~s}, 2 \mathrm{H}, \mathrm{N}-$ $\left.\mathrm{CH}_{2}\right), 6.79\left(\mathrm{~d}, \mathrm{~J}=7.65 \mathrm{~Hz}, 2 \mathrm{H}, 4\right.$-methoxyphenyl- $\left.\mathrm{C}_{2,6}-\mathrm{H}\right)$, 7.25-7.30 (m, 5H, phenyl-H), 7.38 (d, J =7.65 Hz, 2H, 4methoxyphenyl- $\left.\mathrm{C}_{3,5}-\mathrm{H}\right), 7.78\left(\mathrm{~s}, 2 \mathrm{H}, \mathrm{NH}_{2}, \mathrm{D}_{2} \mathrm{O}\right.$ exchangeable), 9.87 (s, 1H, NH, $\mathrm{D}_{2} \mathrm{O}$ exchangeable). Anal. Calcd. for $\mathrm{C}_{25} \mathrm{H}_{22} \mathrm{~N}_{6} \mathrm{O}_{4} \mathrm{~S}_{2 .} 1 / 2 \mathrm{H}_{2} \mathrm{O}$ (543.61): C, 55.19; H, 4.23; N, 15.45. Found: C, 55.25; H, 4.23; N, 15.48.
5- Benzyl-3-methyl-8-\{[2-(4-acetylphenylamino)-2oxoethyl]sulfanyl $\}-4-0 x 0-4,5-d i h y d r o t h i e n o ~[3,2-e]$ $[1,2,4]$ triazolo $[4,3-a]$ pyrimidine-2-carboxamide (7e)

M.P. 250-2 ${ }^{\circ}$; ; Yield 67\%; IR (KBr, $\left.\mathrm{cm}^{-1}\right)$ : 3336, 3173 $\left(\mathrm{NH}_{2}\right), 1675(\mathrm{C}=\mathrm{O}), 1594,1538(\mathrm{C}=\mathrm{N}), 1271,1121(\mathrm{C}-\mathrm{S}-$ C). ${ }^{1} \mathrm{H}-\mathrm{NMR}(\delta \mathrm{ppm}): 2.51\left(\mathrm{~s}, 3 \mathrm{H}, \mathrm{COCH}_{3}\right), 2.74(\mathrm{~s}, 3 \mathrm{H}$, $\left.\mathrm{CH}_{3}\right), 3.99$ (s, 2H, S-CH $), 5.33\left(\mathrm{~s}, 2 \mathrm{H}, \mathrm{N}-\mathrm{CH}_{2}\right), 7.31-7.44$ (m, 5H, phenyl-H), 7.55 (d, J = 8.7 Hz, 2H, 4-acetylphenyl$\left.\mathrm{C}_{3,5}-\mathrm{H}\right), 7.79\left(\mathrm{~s}, 2 \mathrm{H}, \mathrm{NH}_{2}, \mathrm{D}_{2} \mathrm{O}\right.$ exchangeable), 7.87 (d, J $=8.7 \mathrm{~Hz}, 2 \mathrm{H}, 4$-acetylphenyl- $\left.\mathrm{C}_{2,6}-\mathrm{H}\right), 10.38\left(\mathrm{~s}, 1 \mathrm{H}, \mathrm{NH}, \mathrm{D}_{2} \mathrm{O}\right.$ exchangeable). Anal. Calcd. for $\mathrm{C}_{26} \mathrm{H}_{22} \mathrm{~N}_{6} \mathrm{O}_{4} \mathrm{~S}_{2}$ (546.62): $\mathrm{C}$, 57.13; H, 4.06; N, 15.37. Found: C, 57.17; H, 4.11; N, 15.45.

5- Benzyl-3-methyl-8-\{[2-(4-methoxyphenyl)ethylamino)2-oxoethyl]sulfanyl\}-4-oxo-4,5-dihydrothieno[3,2-e] $[1,2,4]$ triazolo $[4,3-a]$ pyrimidine-2-carboxamide (7f)

M.P. $210-2{ }^{\circ} \mathrm{C}$; Yield 67\%; IR $\left(\mathrm{KBr}, \mathrm{cm}^{-1}\right)$ : 3382, 3332 $\left(\mathrm{NH}_{2}\right), 1686(\mathrm{C}=\mathrm{O}), 1594(\mathrm{C}=\mathrm{N}), 1252,1062(\mathrm{C}-\mathrm{S}-\mathrm{C}) .{ }^{1} \mathrm{H}-$ NMR $\left(\delta\right.$ ppm): $2.54\left(\mathrm{t}, \mathrm{J}=7.8 \mathrm{~Hz}, 2 \mathrm{H}, \underline{\mathrm{CH}_{2}} \mathrm{CH}_{2} \mathrm{NH}\right), 2.75$ (s, $\left.3 \mathrm{H}, \mathrm{CH}_{3}\right), 3.15$ (q, J = 7.8 Hz, $\left.2 \mathrm{H}, \mathrm{CH}_{2} \mathrm{CH}_{2} \mathrm{NH}\right), 3.70$ (s, $\left.3 \mathrm{H}, \mathrm{OCH}_{3}\right), 3.73\left(\mathrm{~s}, 2 \mathrm{H}, \mathrm{S}-\mathrm{CH}_{2}\right), 5.33\left(\mathrm{~s}, 2 \mathrm{H}, \mathrm{N}-\mathrm{CH}_{2}\right), 6.82$ $\left(\mathrm{d}, \mathrm{J}=8.7 \mathrm{~Hz}, 2 \mathrm{H}, 4\right.$-methoxyphenyl- $\left.\mathrm{C}_{3}, 5-\mathrm{H}\right), 7.06(\mathrm{~d}, \mathrm{~J}=8.7$ $\mathrm{Hz}, 2 \mathrm{H}$, 4-methoxyphenyl- $\left.\mathrm{C}_{2,6}-\mathrm{H}\right)$, 7.26-7.43 (m, 5H, phenyl-H), 7.83 (s, 2H, $\mathrm{NH}_{2}, \mathrm{D}_{2} \mathrm{O}$ exchangeable), 8.16 (t, J = 5.7 $\mathrm{Hz}, 1 \mathrm{H}, \mathrm{NH}, \mathrm{D}_{2} \mathrm{O}$ exchangeable). ${ }^{13} \mathrm{C}-\mathrm{NMR}$ (d ppm): 14.25

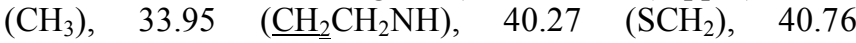
$\left(\mathrm{CH}_{2} \mathrm{CH}_{2} \mathrm{NH}\right), 45.09\left(\mathrm{CH}_{2}\right.$-benzyl), $54.92\left(\mathrm{OCH}_{3}\right), 113.68$ (methoxyphenyl- $\mathrm{C}_{3}$ and $\mathrm{C}_{5}$ ), 118.29 (benzyl- $\mathrm{C}_{4}$ ), 127.49 (thienotriazolopyrimidine- $\mathrm{C}_{2}$ ), 127.85 (benzyl- $\mathrm{C}_{2}$ and $\mathrm{C}_{6}$ ), 128.30 (benzyl- $\mathrm{C}_{3}$ and $\mathrm{C}_{5}$ ), 129.27 (thienotriazolopyrimidine- $\mathrm{C}_{3}$ ), 129.43 (methoxyphenyl- $\mathrm{C}_{2}$ and $\mathrm{C}_{6}$ ), 130.96 (methoxyphenyl- $\mathrm{C}_{1}$ ), 135.79 (thienotriazolopyrimidine- $\mathrm{C}_{3 \mathrm{a}}$ ), 136.58 (thienotriazolopyrimidine- $\mathrm{C}_{8 \mathrm{a}}$ ), 141.25 (thienotriazolopyrimidine- $\mathrm{C}_{8}$ ), 141.88 (benzyl- $\mathrm{C}_{1}$ ), 149.40 (thienotriazolopyrimidine- $\mathrm{C}_{5 \mathrm{a}}$ ), 156.01 (methoxy- $\mathrm{C}_{4}$ ), 157.61 (thienotriazolopyrimidine- $\left.\mathrm{C}_{4}-\mathrm{C}=\mathrm{O}\right), 163.05\left(\mathrm{CONH}_{2}\right), 166.19$ (CO$\mathrm{NH}$ ).Anal. Calcd. for $\mathrm{C}_{27} \mathrm{H}_{26} \mathrm{~N}_{6} \mathrm{O}_{4} \mathrm{~S}_{2}$ (562.66): C, 57.63; H, 4.66; N, 14.94. Found: C, 57.34; H, 4.47; N, 14.71.

\section{5- Benzyl-3-methyl-8-\{[2-(substituted amino)-2- oxoethyl]sulfonyl\}-4-oxo-4,5-dihydrothieno[3,2-e] $[1,2,4]$ triazolo[4,3-a]pyrimidine-2-carboxamides (8a-f)}

An aqueous solution of $4 \%$ potassium permanganate was added dropwise to a stirred solution of the appropriate 7a-f $(0.5 \mathrm{mmol})$ in a mixture of glacial acetic acid and DMF (5 $\mathrm{ml}, 3: 2$ ). The addition of potassium permanganate solution was continued until the purple color persisted for 30 minutes. Stirring was maintained at room temperature for further 2 hours. The reaction mixture was left overnight, then cooled to $5{ }^{\circ} \mathrm{C}$ and saturated sodium sulfite solution was gradually added with stirring until the brown color disappeared. The white precipitate formed was filtered, washed with water, air dried and crystallized from DMF.

\section{5- Benzyl-3-methyl-8-\{[2-(phenylamino)-2- oxoethyl]sulfonyl\}-4-oxo-4,5-dihydrothieno[3,2-e] $[1,2,4]$ triazolo $[4,3-a]$ pyrimidine-2-carboxamide (8a)}

M.P. 268-70 C; Yield 64\%; IR (KBr, $\left.\mathrm{cm}^{-1}\right): 3410,3300$ $\left(\mathrm{NH}_{2}\right), 3252(\mathrm{NH}), 1684(\mathrm{C}=\mathrm{O}), 1588(\mathrm{C}=\mathrm{N}), 1317,1157$ 
$\left(\mathrm{SO}_{2}\right), 1217,1092$ (C-S-C). ${ }^{1} \mathrm{H}-\mathrm{NMR}(\delta \mathrm{ppm}): 2.75$ (s, 3H, $\left.\mathrm{CH}_{3}\right), 3.95$ (s, $\left.2 \mathrm{H}, \mathrm{SO}_{2}-\mathrm{CH}_{2}\right), 5.33\left(\mathrm{~s}, 2 \mathrm{H}, \mathrm{N}-\mathrm{CH}_{2}\right), 7.04-7.43$ (m, $10 \mathrm{H}, 2$ phenyl-H), 7.80 (s, $2 \mathrm{H}, \mathrm{NH}_{2}, \mathrm{D}_{2} \mathrm{O}$ exchangeable), 10.03 (s, 1H, NH, $\mathrm{D}_{2} \mathrm{O}$ exchangeable). Anal. Calcd. for $\mathrm{C}_{24} \mathrm{H}_{20} \mathrm{~N}_{6} \mathrm{O}_{5} \mathrm{~S}_{2}$ (536.58): C, 53.72; H, 3.76; N, 15.66. Found: $\mathrm{C}, 53.59 ; \mathrm{H}, 3.73 ; \mathrm{N} 15.24$.

5- Benzyl-3-methyl-8-\{[2-(4-chlorophenylamino)-2oxoethyl] sulfonyl $\}-4-0 \times 0-4,5$-dihydrothieno $[3,2-e]$ $[1,2,4]$ triazolo $[4,3-a]$ pyrimidine-2-carboxamide $(8 \mathrm{~b})$

M.P. 260-2 C; Yield 65\%; IR ( $\left.\mathrm{KBr}, \mathrm{cm}^{-1}\right): 3420,3300$ $\left(\mathrm{NH}_{2}\right), 3252(\mathrm{NH}), 1686(\mathrm{C}=\mathrm{O}), 1588(\mathrm{C}=\mathrm{N}), 1318,1144$ $\left(\mathrm{SO}_{2}\right), 1216,1092$ (C-S-C). ${ }^{1} \mathrm{H}-\mathrm{NMR}(\delta \mathrm{ppm}): 2.74(\mathrm{~s}, 3 \mathrm{H}$, $\left.\mathrm{CH}_{3}\right), 3.94$ (s, $\left.2 \mathrm{H}, \mathrm{SO}_{2}-\mathrm{CH}_{2}\right), 5.33$ (s, $\left.2 \mathrm{H}, \mathrm{N}-\mathrm{CH}_{2}\right), 7.23-7.45$ (m, $9 \mathrm{H}$, phenyl- $\mathrm{H}$ and 4-chlorophenyl- $\mathrm{H}), 7.81\left(\mathrm{~s}, 2 \mathrm{H}, \mathrm{NH}_{2}\right.$, $\mathrm{D}_{2} \mathrm{O}$ exchangeable), 10.17 (s, $1 \mathrm{H}, \mathrm{NH}, \mathrm{D}_{2} \mathrm{O}$ exchangeable). ${ }^{13} \mathrm{C}-\mathrm{NMR}(\mathrm{d} \mathrm{ppm}): 14.35\left(\mathrm{CH}_{3}\right), 45.39\left(\mathrm{CH}_{2}\right.$-benzyl), 53.67 $\left(\mathrm{SO}_{2} \mathrm{CH}_{2}\right), 114.02$ (chlorophenyl- $\mathrm{C}_{2}$ and $\left.\mathrm{C}_{6}\right), 118.09$ (benzyl$\mathrm{C}_{4}$ ), 127.69(thienotriazolopyrimidine- $\mathrm{C}_{2}$ ), 128.31 (benzyl- $\mathrm{C}_{2}$ and $\mathrm{C}_{6}$ ), 128.61 (benzyl- $\mathrm{C}_{3}$ and $\mathrm{C}_{5}$ ), 129.69 (chlorophenyl- $\mathrm{C}_{3}$ and $\mathrm{C}_{5}$ ), 130.05 (chlorophenyl- $\mathrm{C}_{4}$ ), 130.56 (thienotriazolopyrimidine- $\mathrm{C}_{3}$ ), 135.56 (thienotriazolopyrimidine- $\mathrm{C}_{3 \mathrm{a}}$ ), 137.04 (thienotriazolopyrimidine- $\mathrm{C}_{8 \mathrm{a}}$ ), 140.23 (chlorophenyl- $\mathrm{C}_{1}$ ), 140.94 (thienotriazolopyrimidine- $\mathrm{C}_{8}$ ), 141.97 (benzyl- $\mathrm{C}_{1}$ ), 149.55 (thienotriazolopyrimidine- $\mathrm{C}_{5 \mathrm{a}}$ ), 157.61 (thienotriazolopyrimidine- $\left.\mathrm{C}_{4}-\mathrm{C}=\mathrm{O}\right), 163.05\left(\mathrm{CONH}_{2}\right), 166.19(\mathrm{CONH})$. Anal. Calcd. for $\mathrm{C}_{24} \mathrm{H}_{19} \mathrm{ClN}_{6} \mathrm{O}_{5} \mathrm{~S}_{2}$ (571.03): C,50.48; H, 3.35; N 14.72. Found: C, 50.19; H, 3.12; N, 14.44.

5- Benzyl-3-methyl-8-\{[2-(4-methylphenylamino)-2oxoethyl] sulfonyl\}-4-oxo-4,5-dihydrothieno [3,2-e] $[1,2,4]$ triazolo $[4,3-a]$ pyrimidine-2-carboxamide (8c)

M.P. $272-4{ }^{\circ} \mathrm{C}$; Yield $69 \%$; IR $\left(\mathrm{KBr}, \mathrm{cm}^{-1}\right): 3316,3220$ $\left(\mathrm{NH}_{2}\right), 3260(\mathrm{NH}), 1668,1655(\mathrm{C}=\mathrm{O}), 1592,1559(\mathrm{C}=\mathrm{N})$, 1318, $1145\left(\mathrm{SO}_{2}\right), 1258,1076(\mathrm{C}-\mathrm{S}-\mathrm{C}) .{ }^{1} \mathrm{H}-\mathrm{NMR}(\delta \mathrm{ppm})$ : $2.20\left(\mathrm{~s}, 3 \mathrm{H}, \mathrm{p}\right.$-tolyl- $\left.\mathrm{CH}_{3}\right), 2.70\left(\mathrm{~s}, 3 \mathrm{H}, \mathrm{CH}_{3}\right), 3.88(\mathrm{~s}, 2 \mathrm{H}$, $\left.\mathrm{SO}_{2}-\mathrm{CH}_{2}\right), 5.29\left(\mathrm{~s}, 2 \mathrm{H}, \mathrm{N}-\mathrm{CH}_{2}\right), 7.02(\mathrm{~d}, \mathrm{~J}=7.6 \mathrm{~Hz}, 2 \mathrm{H}, 4-$ tolyl- $\left.\mathrm{C}_{3,5}-\mathrm{H}\right), 7.25-7.39\left(\mathrm{~m}, 7 \mathrm{H}\right.$, phenyl- $\mathrm{H}$ and 4-tolyl- $\mathrm{C}_{2,6}$ $\mathrm{H}), 7.78\left(\mathrm{~s}, 2 \mathrm{H}, \mathrm{NH}_{2}, \mathrm{D}_{2} \mathrm{O}\right.$ exchangeable), $9.92(\mathrm{~s}, 1 \mathrm{H}, \mathrm{NH}$, $\mathrm{D}_{2} \mathrm{O}$ exchangeable). Anal. Calcd. for $\mathrm{C}_{25} \mathrm{H}_{22} \mathrm{~N}_{6} \mathrm{O}_{5} \mathrm{~S}_{2}$ (550.61): C, 54.53; H, 4.03; N 15.26. Found: C, 54.29; H, $3.96 ; \mathrm{N}, 15.14$.

5- Benzyl-3-methyl-8-\{[2-(4-methoxyphenylamino)-2oxoethyl] sulfonyl\}-4-oxo-4,5-dihydrothieno [3,2-e] [1,2,4] triazolo[4,3-a]pyrimidine-2-carboxamide (8d)

M.P. $265-7{ }^{\circ} \mathrm{C}$; Yield $63 \%$; IR $\left(\mathrm{KBr}, \mathrm{cm}^{-1}\right)$ : 3312,3217 $\left(\mathrm{NH}_{2}\right), 3264(\mathrm{NH}), 1678,1655(\mathrm{C}=\mathrm{O}), 1592,1559(\mathrm{C}=\mathrm{N})$, 1318, $1173\left(\mathrm{SO}_{2}\right), 1248,1076(\mathrm{C}-\mathrm{S}-\mathrm{C}) .{ }^{1} \mathrm{H}-\mathrm{NMR}(\delta \mathrm{ppm})$ : $2.75\left(\mathrm{~s}, 3 \mathrm{H}, \mathrm{CH}_{3}\right), 3.71\left(\mathrm{~s}, 3 \mathrm{H}, \mathrm{OCH}_{3}\right), 3.91\left(\mathrm{~s}, 2 \mathrm{H}, \mathrm{SO}_{2}-\right.$ $\left.\mathrm{CH}_{2}\right), 5.33\left(\mathrm{~s}, 2 \mathrm{H}, \mathrm{N}-\mathrm{CH}_{2}\right), 6.83(\mathrm{~d}, \mathrm{~J}=8.7 \mathrm{~Hz}, 2 \mathrm{H}, 4-$ methoxyphenyl- $\left.\mathrm{C}_{2,6}-\mathrm{H}\right), 7.30-7.44(\mathrm{~m}, 7 \mathrm{H}$, phenyl- $\mathrm{H}$ and 4methoxyphenyl- $\left.\mathrm{C}_{3,5}-\mathrm{H}\right), 7.80\left(\mathrm{~s}, 2 \mathrm{H}, \mathrm{NH}_{2}, \mathrm{D}_{2} \mathrm{O}\right.$ exchangeable), $9.90\left(\mathrm{~s}, 1 \mathrm{H}, \mathrm{NH}, \mathrm{D}_{2} \mathrm{O}\right.$ exchangeable). ${ }^{13} \mathrm{C}-\mathrm{NMR}(\mathrm{d}$ ppm): $14.26\left(\mathrm{CH}_{3}\right), 45.19\left(\mathrm{CH}_{2}\right.$-benzyl), $52.27\left(\mathrm{SO}_{2} \mathrm{CH}_{2}\right)$, $55.92\left(\mathrm{OCH}_{3}\right), 114.27$ (methoxyphenyl- $\mathrm{C}_{3}$ and $\left.\mathrm{C}_{5}\right), 118.69$ (benzyl- $\mathrm{C}_{4}$ ), 127.49 (thienotriazolopyrimidine- $\mathrm{C}_{2}$ ), 127.71 (benzyl- $\mathrm{C}_{2}$ and $\mathrm{C}_{6}$ ), 128.29 (benzyl- $\mathrm{C}_{3}$ and $\mathrm{C}_{5}$ ), 129.47 (thienotriazolopyrimidine- $\mathrm{C}_{3}$ ), 129.79 (methoxyphenyl- $\mathrm{C}_{2}$ and $\mathrm{C}_{6}$ ), 131.09 (methoxyphenyl- $\mathrm{C}_{1}$ ), 135.60 (thienotriazolopyrimidine- $\mathrm{C}_{3 \mathrm{a}}$ ), 137.54 (thienotriazolopyrimidine- $\mathrm{C}_{8 \mathrm{a}}$ ),
141.31 (thienotriazolopyrimidine- $\mathrm{C}_{8}$ ), 141.68 (benzyl- $\mathrm{C}_{1}$ ), 149.77 (thienotriazolopyrimidine- $\mathrm{C}_{5 \mathrm{a}}$ ), 155.95 (methoxy- $\mathrm{C}_{4}$ ), 157.81 (thienotriazolopyrimidine- $\left.\mathrm{C}_{4}-\mathrm{C}=\mathrm{O}\right), 162.05 \quad(\mathrm{CO}-$ $\left.\mathrm{NH}_{2}\right), 165.19(\mathrm{CONH}) . \mathrm{MS}, \mathrm{m} / \mathrm{z}$ (relative abundance \%): 566 [M $\left.\mathrm{M}^{\text {H }},(63.87)\right], 488$ (77.31), 478 (65.55), 443 (65.55), 395 (68.91), 380 (68.07), 379 (82.35), 361 (72.27), 352 (70.59), 344 (68.07), 331 (72.27), 313 (78.99), 254 (68.91), 142 (66.39), 119 (79.83), 105 (100), 99 (66.39), 91 (94.96), 69 (72.27). Anal. Calcd. for $\mathrm{C}_{25} \mathrm{H}_{22} \mathrm{~N}_{6} \mathrm{O}_{6} \mathrm{~S}_{2}$ (566.61): C, 52.99 ; H, 3.91; N, 14.83. Found: C, 52.99; H, 4.25; N, 14.92.

\section{5- Benzyl-3-methyl-8-\{[2-(4-acetylphenylamino)-2- oxoethyl]sulfonyl\}-4-oxo-4,5-dihydrothieno [3,2-e] $[1,2,4]$ triazolo $[4,3-a]$ pyrimidine-2-carboxamide (8e)}

M.P. $270-2{ }^{\circ}$ C; Yield 63\%; IR $\left(\mathrm{KBr}, \mathrm{cm}^{-1}\right)$ : 3335,3174 $\left(\mathrm{NH}_{2}\right), 3264(\mathrm{NH}), 1674(\mathrm{C}=\mathrm{O}), 1594(\mathrm{C}=\mathrm{N}), 1359,1176$ $\left(\mathrm{SO}_{2}\right), 1271,1079$ (C-S-C). ${ }^{1} \mathrm{H}-\mathrm{NMR} 2.35\left(\mathrm{~s}, 3 \mathrm{H}, \mathrm{COCH}_{3}\right)$, $2.70\left(\mathrm{~s}, 3 \mathrm{H}, \mathrm{CH}_{3}\right), 3.95\left(\mathrm{~s}, 2 \mathrm{H}, \mathrm{SO}_{2}-\mathrm{CH}_{2}\right), 5.29(\mathrm{~s}, 2 \mathrm{H}, \mathrm{N}-$ $\mathrm{CH}_{2}$ ), 7.29-7.83 (m, 9H, phenyl-H and 4-acetylphenyl-H), $7.96\left(\mathrm{~s}, 2 \mathrm{H}, \mathrm{NH}_{2}, \mathrm{D}_{2} \mathrm{O}\right.$ exchangeable $), 10.48(\mathrm{~s}, 1 \mathrm{H}, \mathrm{NH}$, $\mathrm{D}_{2} \mathrm{O}$ exchangeable). $\mathrm{MS}, \mathrm{m} / \mathrm{z}$ (relative abundance \%): 578 $\left[\mathrm{M}^{\text {H }},(49.45)\right], 566$ (47.25), 373 (47.25), 353 (48.90), 319 (47.80), 306 (51.65), 243 (47.25), 273 (50.55), 235 (45.05), 187 (49.45), 145 (51.65), 135 (46.15), 122 (45.05), 120 (45.05), 118 (56.04), 91 (47.25), 77 (74.18), 60 (100). Anal. Calcd. for $\mathrm{C}_{26} \mathrm{H}_{22} \mathrm{~N}_{6} \mathrm{O}_{6} \mathrm{~S}_{2}(578.62): \mathrm{C}, 53.97 ; \mathrm{H}, 3.83 ; \mathrm{N}$, 14.52. Found: C, 53.89; H, 3.76; N, 14.14 .

5- Benzyl-3-methyl-8-\{[2-(4-methoxyphenyl)ethylamino)2-oxoethyl]sulfonyl\}-4-oxo-4,5-dihydrothieno[3,2-e] $[1,2,4]$ triazolo[4,3-a]pyrimidine-2-carboxamide (8f)

M.P. $280-2{ }^{\circ} \mathrm{C}$; Yield $61 \%$; IR $(\mathrm{KBr}) v_{\max }\left(\mathrm{cm}^{-1}\right)$ : 3330 , $3190\left(\mathrm{NH}_{2}\right), 3274(\mathrm{NH}), 1684(\mathrm{C}=\mathrm{O}), 1594(\mathrm{C}=\mathrm{N}), 1319$, $1145\left(\mathrm{SO}_{2}\right), 1261,1080$ (C-S-C). ${ }^{1} \mathrm{H}-\mathrm{NMR}$ (DMSO-d6): $\delta$ (ppm) $2.53\left(\mathrm{t}, \mathrm{J}=7.8 \mathrm{~Hz}, 2 \mathrm{H}, \mathrm{CH}_{2} \mathrm{CH}_{2} \mathrm{NH}\right), 2.74(\mathrm{~s}, 3 \mathrm{H}$, $\left.\mathrm{CH}_{3}\right), 3.13\left(\mathrm{q}, \mathrm{J}=7.8 \mathrm{~Hz}, 2 \mathrm{H}, \mathrm{CH}_{2} \mathrm{CH}_{2} \mathrm{NH}\right), 3.72(\mathrm{~s}, 3 \mathrm{H}$, $\left.\mathrm{OCH}_{3}\right), 3.79\left(\mathrm{~s}, 2 \mathrm{H}, \mathrm{SO}_{2}-\mathrm{CH}_{2}\right), 5.36\left(\mathrm{~s}, 2 \mathrm{H}, \mathrm{N}-\mathrm{CH}_{2}\right), 6.93(\mathrm{~d}$, $\mathrm{J}=8.7 \mathrm{~Hz}, 2 \mathrm{H}, 4$-methoxyphenyl- $\left.\mathrm{C}_{3,5}-\mathrm{H}\right), 7.15(\mathrm{~d}, \mathrm{~J}=8.7$ $\mathrm{Hz}, 2 \mathrm{H}, 4$-methoxyphenyl- $\left.\mathrm{C}_{2,6}-\mathrm{H}\right), 7.24-7.44(\mathrm{~m}, 5 \mathrm{H}$, phenyl-H), 7.84 (s, 2H, $\mathrm{NH}_{2}, \mathrm{D}_{2} \mathrm{O}$ exchangeable), 8.27 (t, $\mathrm{J}=6.7$ $\mathrm{Hz}, 1 \mathrm{H}, \mathrm{NH}, \mathrm{D}_{2} \mathrm{O}$ exchangeable). Anal. Calcd. (\%) for $\mathrm{C}_{27} \mathrm{H}_{26} \mathrm{~N}_{6} \mathrm{O}_{6} \mathrm{~S}_{2}: \mathrm{C}, 54.53 ; \mathrm{H}, 4.41 ; \mathrm{N}, 14.13$. Found: $\mathrm{C}, 54.29$; $\mathrm{H}, 4.15 ; \mathrm{N}, 14.14$.

\section{5- Benzyl-3-methyl-8-(substituted sulfanyl)-4-oxo-4,5- dihydrothieno $[3,2-e][1,2,4]$ triazolo $[4,3-a]$ pyrimidine-2- carboxamides (9a-c)}

A mixture of $5(0.37 \mathrm{~g}, 1 \mathrm{mmol})$, appropriate alkyl or aralkyl halide $1(1 \mathrm{mmol})$ and anhydrous potassium carbonate $(0.14 \mathrm{~g}, 1 \mathrm{mmol})$ in dry acetone $(20 \mathrm{ml})$ was stirred and refluxed for 5 hours. The mixture was then cooled, poured into ice-cold water, filtered and the solid obtained was dried and crystallized from DMF.

\section{5-Benzyl-3-methyl-8-(methylsulfanyl)-4-oxo-4,5- dihydrothieno $[3,2-e][1,2,4]$ triazolo $[4,3-a]$ pyrimidine-2- carboxamide (9a)}

M.P. 280-2 ${ }^{\circ}$; Y Yield 75\%; IR ( $\left.\mathrm{KBr}, \mathrm{cm}^{-1}\right)$ : 3329, 3149 $\left(\mathrm{NH}_{2}, \mathrm{NH}\right), 1677(\mathrm{C}=\mathrm{O}), 1593,1541(\mathrm{C}=\mathrm{N}), 1288,1029(\mathrm{C}-$ 
S-C). ${ }^{1} \mathrm{H}-\mathrm{NMR}(\delta \mathrm{ppm}): 2.61\left(\mathrm{~s}, 3 \mathrm{H}, \mathrm{CH}_{3}\right), 2.71(\mathrm{~s}, 3 \mathrm{H}, \mathrm{S}-$ $\left.\mathrm{CH}_{3}\right), 5.28\left(\mathrm{~s}, 2 \mathrm{H}, \mathrm{N}-\mathrm{CH}_{2}\right), 7.22-7.40$ (m, 5H, phenyl-H), 7.77 (s, 2H, $\mathrm{NH}_{2}, \mathrm{D}_{2} \mathrm{O}$ exchangeable). Anal. Calcd. for $\mathrm{C}_{17} \mathrm{H}_{15} \mathrm{~N}_{5} \mathrm{O}_{2} \mathrm{~S}_{2}$ (385.46): C, 52.97; H, 3.92; N, 18.17. Found: C, 53.04; H, 3.89; N, 18.31 .

5-Benzyl-3-methyl-8-(ethylsulfanyl)-4-oxo-4,5dihydrothieno $[3,2-e][1,2,4]$ triazolo $[4,3-a]$ pyrimidine-2carboxamide (9b)

M.P. $285-7{ }^{\circ} \mathrm{C}$; Yield 74\%; IR $\left(\mathrm{KBr}, \mathrm{cm}^{-1}\right): 3329,3156$ $\left(\mathrm{NH}_{2}, \mathrm{NH}\right), 1680(\mathrm{C}=\mathrm{O}), 1586,1540(\mathrm{C}=\mathrm{N}), 1268,1060(\mathrm{C}-$ $\mathrm{S}-\mathrm{C}) .{ }^{1} \mathrm{H}-\mathrm{NMR}(\delta \mathrm{ppm}): 1.29\left(\mathrm{t}, \mathrm{J}=7.2 \mathrm{~Hz}, 3 \mathrm{H}, \mathrm{SCH}_{2} \underline{\mathrm{CH}}_{3}\right)$, $2.74\left(\mathrm{~s}, 3 \mathrm{H}, \mathrm{CH}_{3}\right), 3.09$ (q, J = 7.2 Hz, $\left.2 \mathrm{H}, \mathrm{SCH}_{2} \mathrm{CH}_{3}\right), 5.32$ $\left(\mathrm{s}, 2 \mathrm{H}, \mathrm{N}-\mathrm{CH}_{2}\right), 7.26-7.44(\mathrm{~m}, 5 \mathrm{H}$, phenyl-H), $7.77(\mathrm{~s}, 2 \mathrm{H}$, $\mathrm{NH}_{2}, \mathrm{D}_{2} \mathrm{O}$ exchangeable). ${ }^{13} \mathrm{C}-\mathrm{NMR}(\mathrm{d} \mathrm{ppm}): 14.26\left(\mathrm{CH}_{3}\right)$, $14.97\left(\mathrm{SCH}_{2} \mathrm{CH}_{3}\right), 43.45\left(\mathrm{SCH}_{2} \mathrm{CH}_{3}\right), 45.09\left(\mathrm{CH}_{2}\right.$-benzyl), 118.50 (benzyl- $\mathrm{C}_{4}$ ), 127.49 (thienotriazolopyrimidine- $\mathrm{C}_{2}$ ), 127.82 (benzyl- $\mathrm{C}_{2}$ and $\mathrm{C}_{6}$ ), 128.29 (benzyl- $\mathrm{C}_{3}$ and $\mathrm{C}_{5}$ ), 128.97 (thienotriazolopyrimidine- $\mathrm{C}_{3}$ ), 135.92(thienotriazolopyrimidine- $\mathrm{C}_{3 \mathrm{a}}$ ), 136.83 (thienotriazolopyrimidine- $\mathrm{C}_{8 \mathrm{a}}$ ), 140.94 (thienotriazolopyrimidine- $\mathrm{C}_{8}$ ), 141.56 (benzyl- $\mathrm{C}_{1}$ ), 149.58 (thienotriazolopyrimidine- $\mathrm{C}_{5 \mathrm{a}}$ ), 156.18 (thienotriazolopyrimidine- $\left.\mathrm{C}_{4}-\mathrm{C}=\mathrm{O}\right), 163.11\left(\mathrm{CONH}_{2}\right)$. Anal. Calcd. for $\mathrm{C}_{18} \mathrm{H}_{17} \mathrm{~N}_{5} \mathrm{O}_{2} \mathrm{~S}_{2}$ (399.49): C, 54.12; H, 4.29; N, 17.53. Found: C, 54.21; H, 4.34; N, 17.64.

5-Benzyl-3-methyl-8-(benzylsulfanyl)-4-oxo-4,5dihydrothieno $[3,2-e][1,2,4]$ triazolo $[4,3-a]$ pyrimidine-2carboxamide (9c)

M.P. 244-6 ${ }^{\circ} \mathrm{C}$; Yield 77\%; IR $\left(\mathrm{KBr}, \mathrm{cm}^{-1}\right): 3319,3143$ $\left(\mathrm{NH}_{2}, \mathrm{NH}\right), 1682(\mathrm{C}=\mathrm{O}), 1586,1550(\mathrm{C}=\mathrm{N}), 1258,1062(\mathrm{C}-$ S-C). ${ }^{1} \mathrm{H}-\mathrm{NMR}(\delta \mathrm{ppm}): 2.73\left(\mathrm{~s}, 3 \mathrm{H}, \mathrm{CH}_{3}\right), 4.35(\mathrm{~s}, 2 \mathrm{H}, \mathrm{S}-$ $\left.\mathrm{CH}_{2}\right), 5.32\left(\mathrm{~s}, 2 \mathrm{H}, \mathrm{N}-\mathrm{CH}_{2}\right), 7.21-7.42(\mathrm{~m}, 10 \mathrm{H}, 2$ phenyl-H), $7.79\left(\mathrm{~s}, 2 \mathrm{H}, \mathrm{NH}_{2}, \mathrm{D}_{2} \mathrm{O}\right.$ exchangeable). Anal. Calcd. for $\mathrm{C}_{23} \mathrm{H}_{19} \mathrm{~N}_{5} \mathrm{O}_{2} \mathrm{~S}_{2}$ (461.56): C, 59.85; H, 4.15; N, 15.17. Found: 59.91; H, 4.17; N, 15.26.

\section{5-Benzyl-3-methyl-8-(substituted sulfonyl)-4-oxo-4,5- dihydrothieno $[3,2-e][1,2,4]$ triazolo $[4,3-a]$ pyrimidine-2- carboxamides (10a-c)}

An aqueous solution of $4 \%$ potassium permanganate was added dropwise to a stirred solution of the appropriate 9a-c $(5 \mathrm{mmol})$ in glacial acetic acid $(5 \mathrm{ml})$. The addition of potassium permanganate solution was continued until the purple color persisted for 30 minutes. Stirring was maintained at room temperature for further 2 hours. The reaction mixture was left overnight, then cooled to $5{ }^{\circ} \mathrm{C}$, saturated sodium sulfite solution was gradually added with stirring until the brown color disappeared. The white precipitate formed was filtered, washed with water, air dried and crystallized from DMF.

\section{5-Benzyl-3-methyl-8-(methylsulfonyl)-4-oxo-4,5- dihydrothieno $[3,2-e][1,2,4]$ triazolo $[4,3-a]$ pyrimidine-2- carboxamide (10a)}

M.P. $>300^{\circ} \mathrm{C}$; Yield 68\%; IR $\left(\mathrm{KBr}, \mathrm{cm}^{-1}\right): 3374,3183$ $\left(\mathrm{NH}_{2}\right), 3270(\mathrm{NH}), 1681,1640(\mathrm{C}=\mathrm{O}), 1591(\mathrm{C}=\mathrm{N}), 1342$, $1146\left(\mathrm{SO}_{2}\right), 1277,1074(\mathrm{C}-\mathrm{S}-\mathrm{C}) .{ }^{1} \mathrm{H}-\mathrm{NMR}(\delta \mathrm{ppm}): 2.74(\mathrm{~s}$, $\left.3 \mathrm{H}, \mathrm{CH}_{3}\right), 3.68\left(\mathrm{~s}, 3 \mathrm{H}, \mathrm{SO}_{2} \mathrm{CH}_{3}\right), 5.40\left(\mathrm{~s}, 2 \mathrm{H}, \mathrm{N}-\mathrm{CH}_{2}\right), 7.27-$ 7.47 (m, 5H, phenyl-H), $7.87\left(\mathrm{~s}, 2 \mathrm{H}, \mathrm{NH}_{2}, \mathrm{D}_{2} \mathrm{O}\right.$ exchangeable. Anal. Calcd. for $\mathrm{C}_{17} \mathrm{H}_{15} \mathrm{~N}_{5} \mathrm{O}_{4} \mathrm{~S}_{2}$ (417.46): C, 48.91; $\mathrm{H}$, $3.62 ; \mathrm{N}, 16.78$. Found: C, 48.89; H, 3.36; N, 16.44 .

\section{5-Benzyl-3-methyl-8-(ethylsulfonyl)-4-oxo-4,5- dihydrothieno $[3,2-e][1,2,4]$ triazolo $[4,3-a]$ pyrimidine-2- carboxamide (10b)}

M.P. $>300^{\circ} \mathrm{C}$; Yield $62 \%$; IR $\left(\mathrm{KBr}, \mathrm{cm}^{-1}\right): 3341,3171$ $\left(\mathrm{NH}_{2}, \mathrm{NH}\right), 1681(\mathrm{C}=\mathrm{O}), 1586(\mathrm{C}=\mathrm{N}), 1336,1145\left(\mathrm{SO}_{2}\right)$, 1272, $1076(\mathrm{C}-\mathrm{S}-\mathrm{C}) .{ }^{1} \mathrm{H}-\mathrm{NMR}(\delta \mathrm{ppm}): 2.29(\mathrm{t}, \mathrm{J}=7.6 \mathrm{~Hz}$, $\left.3 \mathrm{H}, \mathrm{SO}_{2} \mathrm{CH}_{2} \underline{\mathrm{CH}}_{3}\right), 2.75\left(\mathrm{~s}, 3 \mathrm{H}, \mathrm{CH}_{3}\right), 3.98$ (q, J = $7.6 \mathrm{~Hz}, 2 \mathrm{H}$, $\mathrm{SO}_{2} \mathrm{CH}_{2} \mathrm{CH}_{3}$ ), 5.40 (s, 2H, N-CH $)_{2}, 7.26-7.37$ (m, 5H, phenyl-H), 7.89 (s, 2H, NH, $\mathrm{D}_{2} \mathrm{O}$ exchangeable). ${ }^{13} \mathrm{C}-\mathrm{NMR}(\mathrm{d}$ ppm): $\quad 14.27\left(\mathrm{CH}_{3}\right), \quad 14.80 \quad\left(\mathrm{SO}_{2} \mathrm{CH}_{2} \mathrm{CH}_{3}\right), \quad 29.95$ $\left(\mathrm{SO}_{2} \mathrm{CH}_{2} \mathrm{CH}_{3}\right), 45.09$ ( $\mathrm{CH}_{2}$-benzyl), 118.40 (benzyl- $\left.\mathrm{C}_{4}\right)$, 127.38 (thienotriazolopyrimidine- $\mathrm{C}_{2}$ ), 127.81 (benzyl- $\mathrm{C}_{2}$ and $\mathrm{C}_{6}$ ), 128.26 (benzyl- $\mathrm{C}_{3}$ and $\mathrm{C}_{5}$ ), 128.87 (thienotriazolopyrimidine- $\mathrm{C}_{3}$ ), 135.82 (thienotriazolopyrimidine- $\mathrm{C}_{3 \mathrm{a}}$ ), 136.73 (thienotriazolopyrimidine- $\mathrm{C}_{8 \mathrm{a}}$ ), 140.84 (thienotriazolopyrimidine- $\mathrm{C}_{8}$ ), 141.46 (benzyl- $\mathrm{C}_{1}$ ), 149.48 (thienotriazolopyrimidine- $\mathrm{C}_{5 \mathrm{a}}$ ), 156.08 (thienotriazolopyrimidine- $\mathrm{C}_{4}-\mathrm{C}=\mathrm{O}$ ), $163.01\left(\mathrm{CONH}_{2}\right)$. Anal. Calcd. for $\mathrm{C}_{18} \mathrm{H}_{17} \mathrm{~N}_{5} \mathrm{O}_{4} \mathrm{~S}_{2}$ (431.49): C, 50.10; H, 3.97; N, 16.23. Found: C, 49.89; H, 3.76; N, 16.14 .

\section{5-Benzyl-3-methyl-8-(benzylsulfonyl)-4-oxo-4,5- dihydrothieno $[3,2-e][1,2,4]$ triazolo $[4,3-a]$ pyrimidine-2- carboxamide (10c)}

M.P. 280-2 ${ }^{\circ}$ C; Yield 67\%; IR (KBr, $\left.\mathrm{cm}^{-1}\right)$ : 3376, 3165 $\left(\mathrm{NH}_{2}\right), 3245(\mathrm{NH}), 1684,1640(\mathrm{C}=\mathrm{O}), 1594(\mathrm{C}=\mathrm{N}), 1332$, $1144\left(\mathrm{SO}_{2}\right), 1275,1076$ (C-S-C). ${ }^{1} \mathrm{H}-\mathrm{NMR} 2.74(\mathrm{~s}, 3 \mathrm{H}$, $\left.\mathrm{CH}_{3}\right), 4.46\left(\mathrm{~s}, 2 \mathrm{H}, \mathrm{SO}_{2} \mathrm{CH}_{2}\right), 5.41\left(\mathrm{~s}, 2 \mathrm{H}, \mathrm{N}-\mathrm{CH}_{2}\right), 7.23-7.44$ (m, 10H, 2 phenyl-H), 7.88 (s, 2H, $\mathrm{NH}_{2}, \mathrm{D}_{2} \mathrm{O}$ exchangeable). $\mathrm{MS}, \mathrm{m} / \mathrm{z}$ (relative abundance \%): $493\left[\mathrm{M}^{+1},(5.43)\right], 428$ (22.22), 426 (20.93), 404 (27.13), 374 (23.77), 370 (24.55), 268 (22.22), 251 (23.00), 230 (20.93), 191 (23.00), 172 (22.48), 137(21.19), 91(100), 83 (26.61).Anal. Calcd. for $\mathrm{C}_{23} \mathrm{H}_{19} \mathrm{~N}_{5} \mathrm{O}_{4} \mathrm{~S}_{2}$ (493.56): C, 55.97; H, 3.88; N, 14.19. Found: C, 55.89; H, 3.76; N, 14.04 .

\section{5-Benzyl-3-methyl-8-substituted-4-oxo-4,5- dihydrothieno $[3,2-e][1,2,4]$ triazolo $[4,3-a]$ pyrimidine-2- carboxamides (11a-c)}

A mixture of 9a (1.16 g, $3 \mathrm{mmol})$ and the selected amine (9 mmol) was heated in an oil bath at $160-170 \mathrm{C}$ for $6 \mathrm{~h}$. The reaction mixture was triturated with dilute hydrochloric acid, filtered, washed with water, dried, and crystallized from DMF.

\section{5-Benzyl-3-methyl-8-morpholino-4-oxo-4,5- dihydrothieno[3,2-e $][1,2,4]$ triazolo $[4,3-a]$ pyrimidine-2- carboxamide (11a)}

M.P. 246-8 ${ }^{\circ} \mathrm{C}$; Yield 66\%; IR $\left(\mathrm{KBr}, \mathrm{cm}^{-1}\right)$ : 3357, 3156 $\left(\mathrm{NH}_{2}\right), 1689,1642(\mathrm{C}=\mathrm{O}), 1602(\mathrm{C}=\mathrm{N}), 1164,1075(\mathrm{C}-\mathrm{S}-\mathrm{C})$. ${ }^{1} \mathrm{H}-\mathrm{NMR}(\delta \mathrm{ppm}): 2.06-2.69\left(\mathrm{~m}, 11 \mathrm{H}, \mathrm{CH}_{3}\right.$ and morpholine$\mathrm{H}), 5.29\left(\mathrm{~s}, 2 \mathrm{H}, \mathrm{N}-\mathrm{CH}_{2}\right), 7.20-7.29(\mathrm{~m}, 5 \mathrm{H}$, phenyl-H), 7.59 (s, 2H, $\mathrm{NH}_{2}, \mathrm{D}_{2} \mathrm{O}$ exchangeable). ${ }^{13} \mathrm{C}-\mathrm{NMR}$ (d ppm): 14.19 
$\left(\mathrm{CH}_{3}\right), 14.73$ (morpholin- $\mathrm{C}_{3}$ and $\left.\mathrm{C}_{5}\right), 46.74\left(\mathrm{CH}_{2}\right.$-benzyl), 54.30 (morpholin- $\mathrm{C}_{2}$ and $\mathrm{C}_{6}$ ), 118.93 (benzyl- $\mathrm{C}_{4}$ ), 126.68 (thienotriazolopyrimidine- $\mathrm{C}_{2}$ ), 127.40 (benzyl- $\mathrm{C}_{2}$ and $\mathrm{C}_{6}$ ), 128.43 (benzyl- $\mathrm{C}_{3}$ and $\mathrm{C}_{5}$ ), 128.97 (thienotriazolopyrimidine- $\mathrm{C}_{3}$ ), 135.24 (thienotriazolopyrimidine- $\mathrm{C}_{3 \mathrm{a}}$ ), 137.17 (thienotriazolopyrimidine- $\mathrm{C}_{8 \mathrm{a}}$ ), 140.94 (thienotriazolopyrimidine$\mathrm{C}_{8}$ ), 141.56 (benzyl- $\mathrm{C}_{1}$ ), 149.58 (thienotriazolopyrimidine$\mathrm{C}_{5 \mathrm{a}}$ ), 158.44 (thienotriazolopyrimidine- $\mathrm{C}_{4}-\mathrm{C}=\mathrm{O}$ ), 163.10 $\left(\mathrm{CONH}_{2}\right)$. Anal. Calcd. for $\mathrm{C}_{20} \mathrm{H}_{20} \mathrm{~N}_{6} \mathrm{O}_{3} \mathrm{~S}$ (424.48): C, 56.59; H, 4.75; N, 19.80. Found: C, 56.72; H, 4.74; N, 19.97.

\section{5-Benzyl-3-methyl-8-piperidino-4-oxo-4,5- dihydrothieno $[3,2-e][1,2,4]$ triazolo $[4,3-a]$ pyrimidine-2- carboxamide (11b)}

M.P. 255-7 ${ }^{\circ}$ C; Yield 65\%; IR $\left(\mathrm{KBr}, \mathrm{cm}^{-1}\right): 3358,3166$ $\left(\mathrm{NH}_{2}\right), 1679,1653(\mathrm{C}=\mathrm{O}), 1604(\mathrm{C}=\mathrm{N}), 1174,1021(\mathrm{C}-\mathrm{S}-\mathrm{C})$. ${ }^{1} \mathrm{H}-\mathrm{NMR}(\delta \mathrm{ppm}): 2.50-2.89\left(\mathrm{~m}, 13 \mathrm{H}, \mathrm{CH}_{3}\right.$ and piperidine$\mathrm{H}), 5.30\left(\mathrm{~s}, 2 \mathrm{H}, \mathrm{N}-\mathrm{CH}_{2}\right), 7.22-7.36(\mathrm{~m}, 5 \mathrm{H}$, phenyl-H), 7.60 (s, 2H, $\mathrm{NH}_{2}, \mathrm{D}_{2} \mathrm{O}$ exchangeable). Anal. Calcd. for $\mathrm{C}_{21} \mathrm{H}_{22} \mathrm{~N}_{6} \mathrm{O}_{2} \mathrm{~S}$ (422.50): C, 59.70; H, 5.25; N, 19.89. Found: C, 59.59; H, 5.16; N, 19.74 .

\section{5-Benzyl-3-methyl-8-(N-phenylpiperazino)-4-oxo-4,5- dihydrothieno $[3,2-e][1,2,4]$ triazolo $[4,3-a]$ pyrimidine-2- carboxamide (11c)}

M.P. 251-3 ${ }^{\circ} \mathrm{C}$; Yield 62\%; IR $\left(\mathrm{KBr}, \mathrm{cm}^{-1}\right): 3358,3166$ $\left(\mathrm{NH}_{2}\right), 1689,1642(\mathrm{C}=\mathrm{O}), 1602(\mathrm{C}=\mathrm{N}), 1164,1026(\mathrm{C}-\mathrm{S}-\mathrm{C})$. ${ }^{1} \mathrm{H}-\mathrm{NMR}(\delta \mathrm{ppm}): 2.50-2.89\left(\mathrm{~m}, 11 \mathrm{H}, \mathrm{CH}_{3}\right.$ and piperazine$\mathrm{H}), 5.30\left(\mathrm{~s}, 2 \mathrm{H}, \mathrm{N}-\mathrm{CH}_{2}\right), 7.22-7.36(\mathrm{~m}, 10 \mathrm{H}, 2$ phenyl-H), $7.60\left(\mathrm{~s}, 2 \mathrm{H}, \mathrm{NH}_{2}, \mathrm{D}_{2} \mathrm{O}\right.$ exchangeable). Anal. Calcd. for $\mathrm{C}_{26} \mathrm{H}_{25} \mathrm{~N}_{7} \mathrm{O}_{2} \mathrm{~S}$ (499.59): C, 62.51; H, 5.04; N, 19.63. Found: C, 62.39; H, 4.79; N, 19.34.

\section{BIOLOGICAL EVALUATION}

\section{Anti-Inflammatory (AI) Activity}

\section{Formalin-Induced Paw Edema Bioassay (Acute Inflamma- tory Model)}

Male albino rats weighing 180-200 g were used throughout the assay [19]. They were kept in the animal house under standard condition of light and temperature with free access to food and water. The animals were randomly divided into groups each of five rats. One group of five rats was kept as a control and another group received the standard drug diclofenac $\mathrm{Na}$ (at a dose of $20 \mathrm{mg} / \mathrm{kg}$ body weight po). A solution of formalin $(2 \%, 0.1 \mathrm{~mL})$ was injected into the subplanter region of the left hind paw under light ether anesthesia $1 \mathrm{~h}$ after oral administration (po) of the test compound (at a dose level of $20 \mathrm{mg} / \mathrm{kg}$ body weight). The paw volume (mL) was measured by means of water plethysmometer and remeasured again 1,2 and $4 \mathrm{~h}$ after administration of formalin. The edema was expressed as an increase in the volume of paw, and the percentage of edema inhibition for each rat and each group was obtained as follows:

$\%$ Inhibition $=(\mathrm{Vt}-\mathrm{Vo})$ control- $(\mathrm{Vt}-\mathrm{Vo})$ tested compound / $(\mathrm{Vt}-\mathrm{Vo})$ control $\times 100$

Where $\mathrm{Vt}=$ volume of edema at specific time interval and $\mathrm{Vo}_{\mathrm{o}}=$ volume of edema at zero time interval.
Formalin-Induced Paw Edema Bioassay (Sub-Acute Inflammatory Model)

Rats in the first experiment were given the same test compounds at a dose level of $20 \mathrm{mg} / \mathrm{kg}$ body weight daily for 7 consecutive days. A solution of formalin $(2 \%, 0.1 \mathrm{~mL})$ was injected into the subplanter region of the left hind paw under light ether anesthesia $1 \mathrm{~h}$ after oral administration (po) of the test compound. A second injection of formalin $(2 \%$, $0.1 \mathrm{~mL}$ ) was given on the third day. The changes in the volume of paw were measured plethymographically at the first and eighth days [19].

\section{Ulcerogenic Activity}

Male albino rats (180-200 g) were divided into groups each of five animals and were fasted for $12 \mathrm{~h}$ prior to the administration of the test compounds. Water was given ad libitum. Control group received $1 \%$ gum acacia orally. Other groups received diclofenac $\mathrm{Na}$ or the test compounds orally in two equal doses at 0 and $12 \mathrm{~h}$ for three successive days at a dose of $300 \mathrm{mg} / \mathrm{kg}$ per day. Animals were sacrificed by diethyl ether $6 \mathrm{~h}$ after the last dose and their stomachs were removed. An opening at the greater curvature was made and the stomach was cleaned by washing with cold saline and inspected with a $3 \times$ magnifying lens for any evidence of hyperemia, hemorrhage, definite hemorrhagic erosion or ulcer [20].

\section{Acute Toxicity}

Twelve groups of rats (180-200 g) each consists of five animals, were used in this test [21]. The animals were fasted for $24 \mathrm{~h}$ prior to administration of the test compounds. The compounds were given orally in graded doses of $0.1-0.3$ $\mathrm{g} / \mathrm{kg}$ body weight, po. The compounds were screened at graded doses for their acute lethal doses $\left(\mathrm{ALD}_{50}\right)$ and the mortalities were recorded at each dose level after $24 \mathrm{~h}$.

\section{Determination of Effective Dose $50\left(\right.$ ED $\left._{50}\right)$}

The selected compounds were further tested at 5, 10, 20, 40 , and $50 \mathrm{mg} / \mathrm{Kg}$ body weight and the $\mathrm{ED}_{50}$ was determined by measuring the inhibition of the edema volume $2 \mathrm{~h}$ after formalin injection.

\section{Statistical Analysis of Data}

The data obtained are presented as means \pm SE of the mean. The concentration- dependent effects of various drugs in vitro were evaluated statistically by the randomized block design analysis of Variance (ANOVA) followed by StudentNewman-Keuls Multiple Comparison Test. The difference in results was considered significant when $\mathrm{P}<0.05$.

\section{Analgesic Activity}

Analgesic activity was determined using tail withdrawal response to immersion of rat tail in water at $55{ }^{\circ} \mathrm{C}$ according to the procedure described by Janssen et al. [22]. Male albino rats weighing 120-150 g were used throughout this assay. They were kept in the animal house under standard condition of light and temperature with free access to food and water. The animals were randomly divided into groups 
each of five rats. One group of five rats was kept as a control and another group received the standard drug diclofenac $\mathrm{Na}$ (at a dose of $20 \mathrm{mg} / \mathrm{kg}$ body weight po). The tested compounds were administrated orally at a dose of $20 \mathrm{mg} / \mathrm{kg}$ and diclofenac $\mathrm{Na}$ was used as a reference drug $(20 \mathrm{mg} / \mathrm{kg})$. The recorded values were the average of five administrations \pm SE and the percentage increase of the reaction time (after 1$4 \mathrm{~h}$ time intervals) was calculated in comparison with the basal values.

\section{Docking Studies}

Computer simulated docking experiments were carried out under an MMFF94X force field in (PDB ID code: 1PXX, Diclofenac Na bound in the active site) using chemical computing group's Molecular Operating Environment (MOE-Dock 2008) software, Montréal, Canada [23].

Crystal coordinates from the X-ray crystal structure of COX-2 were taken from the protein data bank (PDB ID code: 1PXX). The ligand molecules were constructed using the builder module and were energy minimized using Force Field MMFF94x. The active site of COX-2 was generated using the MOE-Alpha Site Finder, and then ligands were docked within this active site using the MOE Dock. The lowest energy conformation was selected and the ligand interactions (hydrogen bonding and hydrophobic interaction) with COX-2 were determined.

\section{RESULTS AND DISCUSSION}

\section{Chemistry}

The synthetic pathway of the intermediate and final compounds are illustrated in Schemes $\mathbf{1}$ and 2. According to Scheme1 The key intermediate 5-benzyl-3-methyl4-oxo-8-thioxo-4,5,7,8-tetrahydrothieno[3,2-e][1,2,4] triazolo [4,3-a]pyrimidine-2-carboxamide (5) was prepared through a 4-step synthetic protocol in a good yield according to our reported procedures [17]. 2-chloro-N-substitutedacetamides (6a-f) were prepared by treating different amines with chloroacetyl chloride in methylene chloride and anhydrous $\mathrm{K}_{2} \mathrm{CO}_{3}$, following Baraldi's procedure [24] and was allowed to react with (5) in dry acetone and anhydrous $\mathrm{K}_{2} \mathrm{CO}_{3}$, to give compounds (7a-f). ${ }^{1} \mathrm{H}$ NMR spectra showed a characteristic singlet at 3.73-3.99 ppm integrated for $\mathrm{SCH}_{2}$ protons in addition to $\mathrm{D}_{2} \mathrm{O}$ exchangeable singlet at 8.16-10.38 attributed to $\mathrm{NH}$ proton. Additionally, ${ }^{13} \mathrm{C}$ NMR spectrum for (7f) showed signals at $14.25,40.27,45.09,141.25,157.61$, $163.05,166.19$ due to $\mathrm{CH}_{3}, \mathrm{SCH}_{2}, \mathrm{CH}_{2}$-benzyl, thienotriazolopyrimidine $-\mathrm{C}_{8}$, and the three $\mathrm{C}=\mathrm{O}$ groups respectively providing a substantial proof for the assigned structure of (7a-f) compounds.

Oxidation of the thio function in compounds (7a-f) to the corresponding sulfone derivatives (8a-f) was accomplished using $\mathrm{KMnO}_{4}$ in a mixture of glacial acetic acid and DMF utilizing a previously reported procedure [25]. Analytical and spectral data of compounds (8a-f) are in accordance with their structures. IR spectra of compounds $(\mathbf{8 a - f})$ revealed additional absorption bands at 1317-1359 and 1144-1176 $\mathrm{cm}^{-1}$ corresponding to the sulfonic group. The MS spectra of (8d) and (8e) showed a molecular ion peak at $\mathrm{m} / \mathrm{z} 566$ $(63.87 \%)$ and $578(49.45 \%)$ respectively that matches their molecular formulae. Additionally, ${ }^{13} \mathrm{C}$ NMR spectra of compounds $(\mathbf{8 b})$ and $(\mathbf{8 d})$ revealed signals characteristic to $\mathrm{CH}_{3}$, $\mathrm{SO}_{2} \mathrm{CH}_{2}, \mathrm{CH}_{2}$-benzyl, thienotriazolopyrimidine- $\mathrm{C}_{8}$, and the three $\mathrm{C}=\mathrm{O}$ at their expected chemical shifts.

According to Scheme 2, reaction of (5) with different alkyl and aralkyl derivatives in dry acetone and anhydrous $\mathrm{K}_{2} \mathrm{CO}_{3}$, afforded the target S- alkyl and aralkylthio derivatives (9a-c) IR spectra of these compounds exhibited strong absorption bands at 3319-3329, 3143-3156 and 1677-1682 $\mathrm{cm}^{-1}$ due to $\mathrm{NH}_{2}, \mathrm{NH}$ and $\mathrm{C}=\mathrm{O}$ groups respectively. While their ${ }^{1} \mathrm{H}$ NMR spectra matched their proposed structures. ${ }^{13} \mathrm{C}$ NMR spectra of compound (9b) revealed signals at 14.26 , $14.97,43.45,140.94,156.18,163.11 \mathrm{ppm}$ due to $\mathrm{CH}_{3}$, $\mathrm{SCH}_{2} \mathrm{CH}_{3}, \mathrm{SCH}_{2} \mathrm{CH}_{3}$, thienotriazolopyrimidine- $\mathrm{C}_{8}$, and the two $\mathrm{C}=\mathrm{O}$ respectively.

Synthesis of their corresponding sulfones (10a-c) was accomplished by oxidation of the alkyl or aralkyl thio derivatives (9a-c) using $\mathrm{KMnO}_{4}$ in glacial acetic acid. The chemical structure of these compounds was confirmed by their IR, ${ }^{1} \mathrm{H}$ NMR, ${ }^{13} \mathrm{C}$ NMR and MS spectral data. The IR spectra revealed an additional absorption bands at 1332-1344 and $1142-1146 \mathrm{~cm}^{-1}$ due to $\mathrm{SO}_{2}$ group, while their ${ }^{1} \mathrm{H}$ NMR and ${ }^{13} \mathrm{C}$ NMR spectra matched their proposed structures. Mass spectrum of (10c) showed a molecular ion peak at $\mathrm{m} / \mathrm{z} 493$ $(5.43 \%)$ which is in accordance with its molecular formula.

Moreover, fusion of the methylthio derivative (9a) with the appropriate aromatic amines in an oil bath at $160-170^{\circ} \mathrm{C}$ afforded the corresponding 8-amino derivatives (11a-c). ${ }^{1} \mathrm{H}$ NMR showed the absence of the methylthio singlet and the appearance of the signals assigned to the morpholine, piperidine and piperazine protons as well as ${ }^{13} \mathrm{C}-\mathrm{NMR}$ spectrum for compound (11a) showed signals at 14.19, 14.73, $54.30,140.94,158.44,163.10 \mathrm{ppm}$ due to $\mathrm{CH}_{3}$, morpholine $\mathrm{C}_{3}$ and $\mathrm{C}_{5}$, morpholine $\mathrm{C}_{2}$ and $\mathrm{C}_{6}$, thienotriazolopyrimidine$\mathrm{C}_{8}$, and the two $\mathrm{C}=\mathrm{O}$ respectively which provided further confirmation of the structure.

\section{BIOLOGICAL EVALUATION}

\section{Anti-Inflammatory (AI) Activity}

The AI activity of the designed compounds was evaluated by utilizing the formalin-induced paw edema bioassay [19] using diclofenac $\mathrm{Na}(20 \mathrm{mg} / \mathrm{kg})$ as a reference standard anti-inflammatory agent. The paw edema was employed as a model for acute and sub-acute inflammation. The data obtained were presented in (Tables $\mathbf{1}$ and 2) and expressed as means \pm SE. Statistical differences of control and test groups was carried out using the Analysis of Variance (ANOVA) followed by 'Student-Newman-Keuls Multiple Comparison Test'. They were performed using computer package of the Statistical Analysis System (SAS, 1987), SAS Incorporation Institute. The difference in results was considered significant when $\mathrm{P}<0.05$.

\section{Formalin-Induced Paw Edema Bioassay (Acute Inflamma- tory Model)}

In this acute inflammatory model [19] each test compound was dosed orally ( $20 \mathrm{mg} / \mathrm{kg}$ body weight) $1 \mathrm{~h}$ prior to induction of inflammation by formalin injection. Diclofenac $\mathrm{Na}$ was utilized as a reference drug at a dose of 


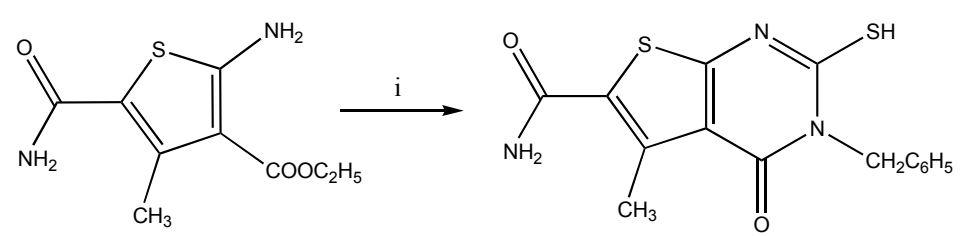

1

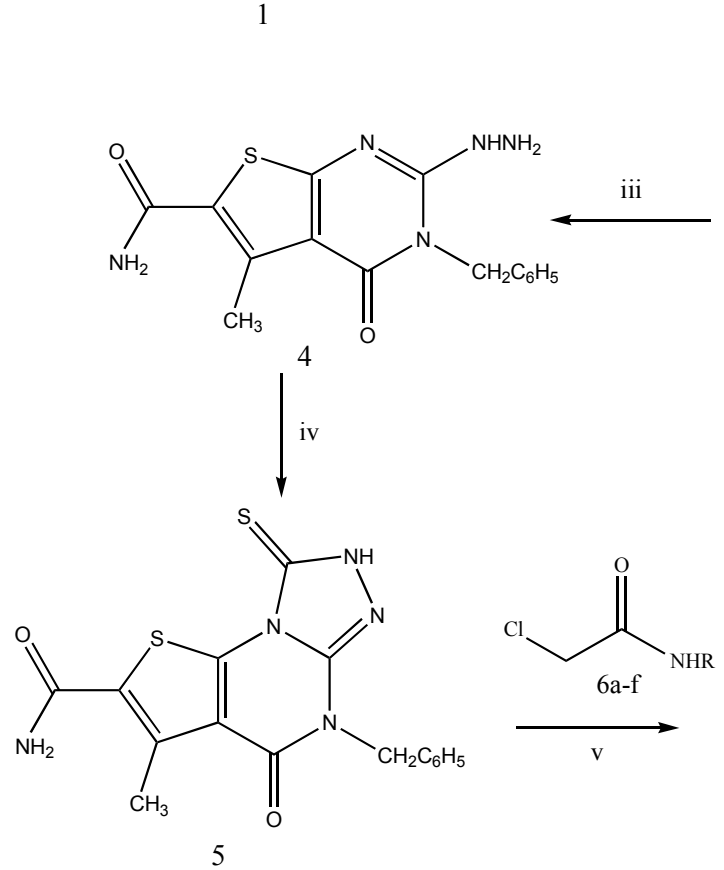

2

$\downarrow$ ii
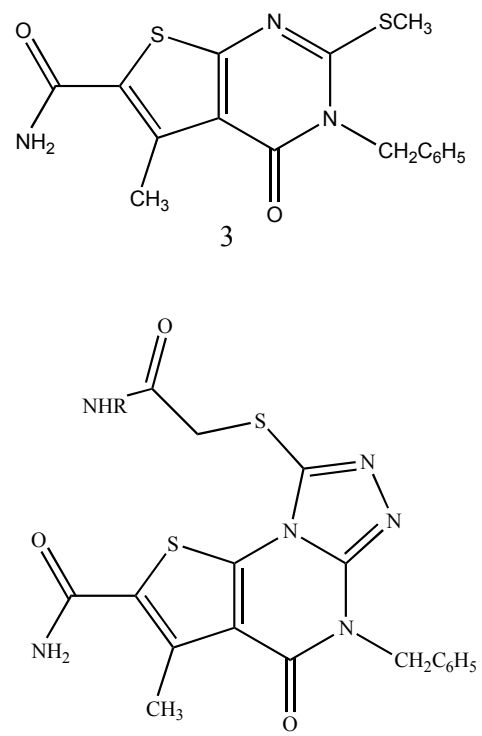

$7 \mathrm{a}-\mathrm{f}$<smiles>CCCCCCCCn1c(=O)c2c(C)c(C(N)=O)sc2n2c(S(=O)(=O)CC(N)=O)nnc12</smiles>

a, $\mathrm{R}=\mathrm{C}_{6} \mathrm{H}_{5} ; \mathrm{b}, \mathrm{R}=4-\mathrm{Cl}-\mathrm{C}_{6} \mathrm{H}_{4} ; \mathrm{c}, \mathrm{R}=4-\mathrm{CH}_{3} \mathrm{C}_{6} \mathrm{H}_{4} ; \mathrm{d}, \mathrm{R}=4-\mathrm{OCH}_{3}-\mathrm{C}_{6} \mathrm{H}_{4} ; \mathrm{e}, \mathrm{R}=4-\mathrm{COCH}_{3} \mathrm{C}_{6} \mathrm{H}_{4} ; \mathrm{f}, \mathrm{R}=4$
Reagents and conditions: i) $\mathrm{C}_{6} \mathrm{H}_{5} \mathrm{CH}_{2} \mathrm{NCS} / \mathrm{K}_{2} \mathrm{CO}_{3} /$ acetonitrile $/$ reflux
ii) $\mathrm{CH}_{3} \mathrm{I} / \mathrm{K}_{2} \mathrm{CO}_{3} /$ dry acetone / reflux ; iii ) $\mathrm{N}_{2} \mathrm{H}_{4} 99 \% / \mathrm{EtOH} /$ reflux; iv) $\mathrm{CS}_{2} / \mathrm{KOH} / \mathrm{EtOH} /$ reflux
v) anhydrous $\mathrm{K}_{2} \mathrm{CO}_{3} /$ dry acetone / reflux; vi) $\mathrm{KMnO}_{4} / \mathrm{gl} . \mathrm{CH}_{3} \mathrm{COOH} / \mathrm{DMF} / \mathrm{RT}$

Scheme 1. Synthesis of compounds 2-5,7a-f and 8a-f.

$20 \mathrm{mg} / \mathrm{kg}$, po. The anti-inflammatory activity was then calculated 1-4 h after induction and presented in (Table 1) as the mean paw volume $(\mathrm{mL})$ in addition to the percentage anti-inflammatory activity (AI\%).

Comparative study of the anti-inflammatory activity of test compounds relative to the reference drug Diclofenac $\mathrm{Na}$ at different time intervals revealed the following:

After $1 \mathrm{~h}$, eight compounds showed distinctive pharmacokinetic profiles as shown from their rapid onset of action which was higher than diclofenac $\mathrm{Na}$ at a dose of $20 \mathrm{mg} / \mathrm{kg}$, po. A remarkable $\mathrm{AI} \%$ was recorded for the thienotriazolopyrimidines $7 \mathbf{f}(61 \%)$, 8a (50\%), 8e (53\%), 8f (76\%), 9a
$(53 \%), 9 c(53 \%), \mathbf{1 0 a}(69 \%)$ and 10c $(73 \%)$, when compared to diclofenac $\mathrm{Na}(42 \%)$, whereas, $7 \mathbf{a}, 8 \mathbf{b}, \mathbf{8 c}, \mathbf{8 d}, \mathbf{1 0 b}$ and 11c showed $\mathrm{AI} \%$ (42-46\%) nearly equipotent to diclofenac $\mathrm{Na}$. The other compounds showed moderate to weak activity. After $2 \mathrm{~h}$ interval, thienotriazolopyrimidines 7f, 8c, 10c, 11a and 11c displayed equipotent anti-inflammatory activity (41$45 \%)$, to diclofenac sodium ( $45 \%)$.

After $4 \mathrm{~h}$ time interval thienotriazolopyrimidines 11a and 11c were nearly effective in inhibiting the paw edema with percentage activity of 64 and $60 \%$ respectively compared to the reference drug $(66 \%)$. while the 2methylthienotriazolopyrimidine $\mathbf{8 f}$ was found to be equipotent to diclofenac sodium, whereas, 7f, 8e, 10a and 10c 
<smiles>CCCCCCCn1c(=O)c2c(C)c(C(N)=O)sc2n2c(=S)[nH]nc12</smiles>

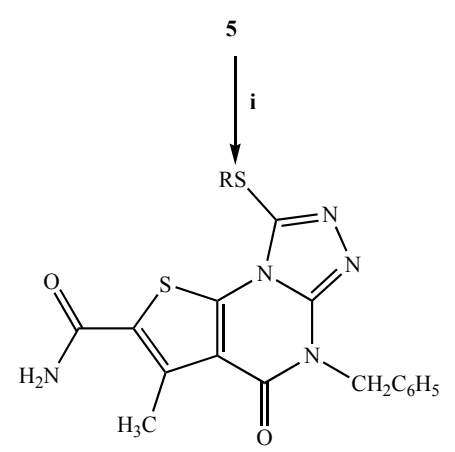

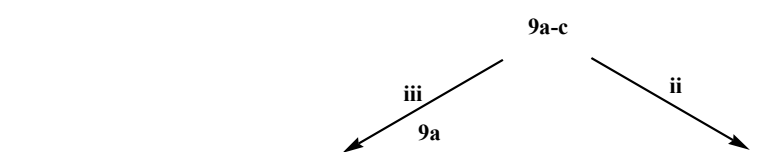

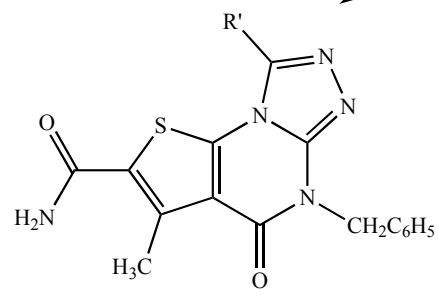

11a-c

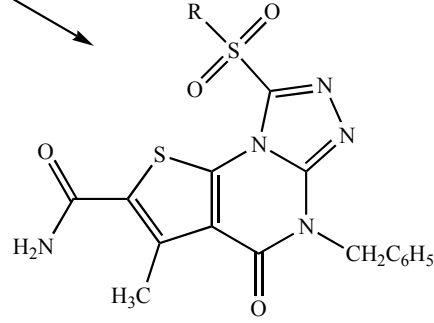

10a-c

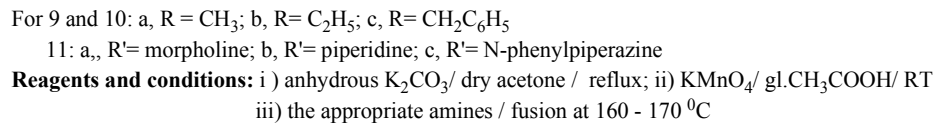

Scheme 2. Synthesis of compounds 9a-c, 10a-c and 11a-c.

displayed lower anti-inflammatory activity (50-52\%). The other compounds showed weak activity.

The most active compounds (7f, 8f, 10a, 10c, 11a and 11c) were further tested at $5,10,20,40$ and $50 \mathrm{mg} / \mathrm{kg}$ body weight in order to determine their $\mathrm{ED}_{50}$ values. All compounds were found to be nearly equipotent $\left(\mathrm{ED}_{50}=14.78\right.$ $19.65 \mathrm{mg} / \mathrm{kg}$, Table 1) compared to the reference drug diclofenac sodium $\left(\mathrm{ED}_{50}=13.56 \mathrm{mg} / \mathrm{kg}\right)$. Results are illustrated by (Fig. 3).

\section{Formalin-Induced Paw Edema Bioassay (Sub-Acute In- flammatory Model)}

For this sub-acute inflammatory model [19], inflammation was induced by formalin injection in the first and third days, and test compounds were administered once orally (at $20 \mathrm{mg} / \mathrm{kg}$ daily) for 7 days. Again, diclofenac sodium was used as a reference agent. The anti-inflammatory activity was calculated at $1^{\text {st }}$ and $8^{\text {th }}$ day after induction and presented in (Table 2) as the mean paw volume and the percentage anti-inflammatory activity (AI\%).
The obtained data revealed that, at $1^{\text {st }}$ day, thienotriazolopyrimidines 10c and 11c displayed anti-inflammatory activity (57\%) nearly equivalent to the reference drug diclofenac sodium (60\%). Meanwhile, 7e and 11a displayed a reasonable anti-inflammatory activity (52 and 51\%) respectively.

At the $8^{\text {th }}$ day, compounds $8 f, 10 a, 10 \mathrm{c}$ and 11c were found to be nearly as effective (54 - 58\%) as diclofenac sodium $(56 \%)$. The other compounds displayed moderate to weak activity. Results are illustrated by (Fig. 4).

A further interpretation of the anti-inflammatory activity of the test compounds in pre-mentioned screens (Tables $\mathbf{1 , 2}$ ) revealed that thienotriazolopyrimidines $8 \mathrm{f}, 10 \mathrm{a}, 10 \mathrm{c}$ and 11c showed remarkable activity in both the acute and subacute inflammatory models. On the other hand, thienotriazolopyrimidines 7f, 8a, 8e, 9a and 9c displayed pronounced activity in the acute inflammatory model nevertheless they proved to be less active in the subacute inflammatory model. This fact would suggest that such type of compounds might be effective in managing acute inflammation, while they 
Table 1. Anti-Inflammatory Activity (AI) of The newly Synthesized Compounds in Formalin-Induced Rat Paw Edema Bioassay (Acute Inflammatory Model)

\begin{tabular}{|c|c|c|c|c|c|}
\hline \multirow{2}{*}{ Compound $^{a}$} & \multicolumn{4}{|c|}{ Volume of edema $(\mathrm{mL})^{b}$} & \multirow{2}{*}{$\begin{array}{c}\mathbf{E D}_{50} \\
(\mathrm{mg} / \mathrm{Kg})\end{array}$} \\
\hline & $\mathbf{0}$ & $1 \mathrm{~h}$ & $2 \mathrm{~h}$ & $4 \mathrm{~h}$ & \\
\hline Control & $0.30 \pm 0.01$ & $0.56 \pm 0.02$ & $0.61 \pm 0.01$ & $0.80 \pm 0.02$ & \\
\hline $7 \mathbf{a}$ & $0.36 \pm 0.01$ & $\begin{array}{c}0.50 \pm 0.02^{c} \\
(46)^{d}\end{array}$ & $\begin{array}{c}0.56 \pm 0.02^{c} \\
(35)\end{array}$ & $\begin{array}{c}0.64 \pm 0.03^{c} \\
(44)\end{array}$ & \\
\hline $7 \mathbf{b}$ & $0.31 \pm 0.01$ & $\begin{array}{c}0.54 \pm 0.01^{c} \\
\quad(11)\end{array}$ & $\begin{array}{c}0.51 \pm 0.01^{c} \\
(35)\end{array}$ & $\begin{array}{c}0.67 \pm 0.01^{c} \\
\quad(28)\end{array}$ & \\
\hline $7 c$ & $0.40 \pm 0.03$ & $\begin{array}{c}0.56 \pm 0.03^{c} \\
(38)\end{array}$ & $\begin{array}{c}0.56 \pm 0.03^{c} \\
(32)\end{array}$ & $\begin{array}{c}0.70 \pm 0.02^{c} \\
(30)\end{array}$ & \\
\hline $7 d$ & $0.33 \pm 0.02$ & $\begin{array}{c}0.56 \pm 0.01^{c} \\
(11)\end{array}$ & $\begin{array}{c}0.60 \pm 0.02^{c} \\
(06)\end{array}$ & $\begin{array}{c}0.69 \pm 0.02^{c} \\
(28)\end{array}$ & \\
\hline $7 e$ & $0.31 \pm 0.03$ & $\begin{array}{c}0.49 \pm 0.01^{c} \\
(30)\end{array}$ & $\begin{array}{c}0.53 \pm 0.01^{c} \\
(29)\end{array}$ & $\begin{array}{c}0.65 \pm 0.03^{c} \\
(52)\end{array}$ & \\
\hline $7 f$ & $0.36 \pm 0.01$ & $\begin{array}{c}0.46 \pm 0.02^{c} \\
(61)\end{array}$ & $\begin{array}{c}0.53 \pm 0.01^{c} \\
(45)\end{array}$ & $\begin{array}{c}0.60 \pm 0.01^{c} \\
(52)\end{array}$ & 18.32 \\
\hline $\mathbf{8 a}$ & $0.33 \pm 0.02$ & $\begin{array}{c}0.46 \pm 0.03^{c} \\
(50)\end{array}$ & $\begin{array}{c}0.52 \pm 0.03^{c} \\
\text { (38) }\end{array}$ & $\begin{array}{c}0.62 \pm 0.02^{c} \\
(42)\end{array}$ & \\
\hline $8 b$ & $0.31 \pm 0.03$ & $\begin{array}{c}0.46 \pm 0.01^{c} \\
(42)\end{array}$ & $\begin{array}{c}0.51 \pm 0.01^{c} \\
(35)\end{array}$ & $\begin{array}{c}0.63 \pm 0.03^{c} \\
(36)\end{array}$ & \\
\hline $8 c$ & $0.35 \pm 0.01$ & $\begin{array}{c}0.49 \pm 0.01^{c} \\
(46)\end{array}$ & $\begin{array}{c}0.58 \pm 0.01^{c} \\
(41)\end{array}$ & $\begin{array}{c}0.69 \pm 0.01^{c} \\
(42)\end{array}$ & \\
\hline $8 d$ & $0.34 \pm 0.01$ & $\begin{array}{c}0.48 \pm 0.02^{c} \\
(46)\end{array}$ & $\begin{array}{c}0.54 \pm 0.02^{c} \\
(35)\end{array}$ & $\begin{array}{c}0.65 \pm 0.01^{c} \\
(38)\end{array}$ & \\
\hline $8 e$ & $0.37 \pm 0.02$ & $\begin{array}{c}0.49 \pm 0.01^{c} \\
(53)\end{array}$ & $\begin{array}{c}0.57 \pm 0.02^{c} \\
(35)\end{array}$ & $\begin{array}{c}0.62 \pm 0.01^{c} \\
(50)\end{array}$ & \\
\hline $8 f$ & $0.33 \pm 0.01$ & $\begin{array}{c}0.39 \pm 0.01^{c} \\
(76)\end{array}$ & $\begin{array}{c}0.52 \pm 0.01^{c} \\
(38)\end{array}$ & $\begin{array}{c}0.50 \pm 0.03^{c} \\
(66)\end{array}$ & 14.78 \\
\hline $9 \mathbf{a}$ & $0.30 \pm 0.02$ & $\begin{array}{c}0.42 \pm 0.01^{c} \\
(53)\end{array}$ & $\begin{array}{c}0.57 \pm 0.03^{c} \\
(12)\end{array}$ & $\begin{array}{c}0.61 \pm 0.02^{c} \\
\text { (38) }\end{array}$ & \\
\hline $9 b$ & $0.32 \pm 0.02$ & $\begin{array}{c}0.52 \pm 0.01^{c} \\
(23)\end{array}$ & $\begin{array}{c}0.55 \pm 0.03^{c} \\
(25)\end{array}$ & $\begin{array}{c}0.62 \pm 0.01^{c} \\
\quad(40)\end{array}$ & \\
\hline $9 \mathrm{c}$ & $0.32 \pm 0.02$ & $\begin{array}{c}0.44 \pm 0.01^{c} \\
\quad(53)\end{array}$ & $\begin{array}{c}0.56 \pm 0.01^{c} \\
(22)\end{array}$ & $\begin{array}{c}0.63 \pm 0.01^{c} \\
(38)\end{array}$ & \\
\hline $10 \mathbf{a}$ & $0.39 \pm 0.01$ & $\begin{array}{c}0.47 \pm 0.01^{c} \\
(69)\end{array}$ & $\begin{array}{c}0.58 \pm 0.03^{c} \\
(38)\end{array}$ & $\begin{array}{c}0.63 \pm 0.01^{c} \\
(52)\end{array}$ & 19.65 \\
\hline $10 b$ & $0.33 \pm 0.02$ & $\begin{array}{c}0.48 \pm 0.02^{c} \\
(42)\end{array}$ & $\begin{array}{c}0.52 \pm 0.01^{c} \\
(38)\end{array}$ & $\begin{array}{c}0.66 \pm 0.01^{c} \\
(34)\end{array}$ & \\
\hline $10 \mathrm{c}$ & $0.37 \pm 0.01$ & $\begin{array}{c}0.44 \pm 0.01^{c} \\
(73)\end{array}$ & $\begin{array}{c}0.54 \pm 0.01^{c} \\
(45)\end{array}$ & $\begin{array}{c}0.62 \pm 0.02^{c} \\
\quad(50)\end{array}$ & 15.63 \\
\hline $11 \mathrm{a}$ & $0.34 \pm 0.02$ & $\begin{array}{c}0.52 \pm 0.03^{c} \\
(30)\end{array}$ & $\begin{array}{c}0.51 \pm 0.02^{c} \\
(45)\end{array}$ & $\begin{array}{c}0.52 \pm 0.01^{c} \\
(64)\end{array}$ & 16.50 \\
\hline $11 b$ & $0.40 \pm 0.02$ & $\begin{array}{c}0.62 \pm 0.01^{c} \\
\quad(15)\end{array}$ & $\begin{array}{c}0.67 \pm 0.01^{c} \\
(12)\end{array}$ & $\begin{array}{c}0.72 \pm 0.02^{c} \\
(36)\end{array}$ & \\
\hline $11 \mathrm{c}$ & $0.38 \pm 0.01$ & $\begin{array}{c}0.53 \pm 0.02^{c} \\
(42)\end{array}$ & $\begin{array}{c}0.56 \pm 0.02^{c} \\
(41)\end{array}$ & $\begin{array}{c}0.58 \pm 0.01^{c} \\
(60)\end{array}$ & 15.74 \\
\hline Diclofenac Na & $0.37 \pm 0.02$ & $\begin{array}{c}0.52 \pm 0.01^{c} \\
(42)\end{array}$ & $\begin{array}{c}0.54 \pm 0.01^{c} \\
(45)\end{array}$ & $\begin{array}{c}0.52 \pm 0.02^{c} \\
(66)\end{array}$ & 13.56 \\
\hline
\end{tabular}

${ }^{\text {a }}$ Dose levels, po: test compounds ( $20 \mathrm{mg} / \mathrm{kg}$ b.wt.), diclofenac $\mathrm{Na}(20 \mathrm{mg} / \mathrm{kg}$ b.wt.)

${ }^{\mathrm{b}}$ Values are expressed as mean \pm S.E. (Number of animals $\mathrm{N}=5$ rats).

${ }^{c}$ Significantly different compared to corresponding control.P $\leq 0.05$.

${ }^{\mathrm{d}}$ Between parentheses (Percentage anti-inflammatory activity, AI \%). 
Table 2. Anti-Inflammatory Activity (AI) of the Newly Synthesized Compounds in Formalin-Induced Rat Paw Edema Bioassay (Sub-Acute Inflammatory Model)

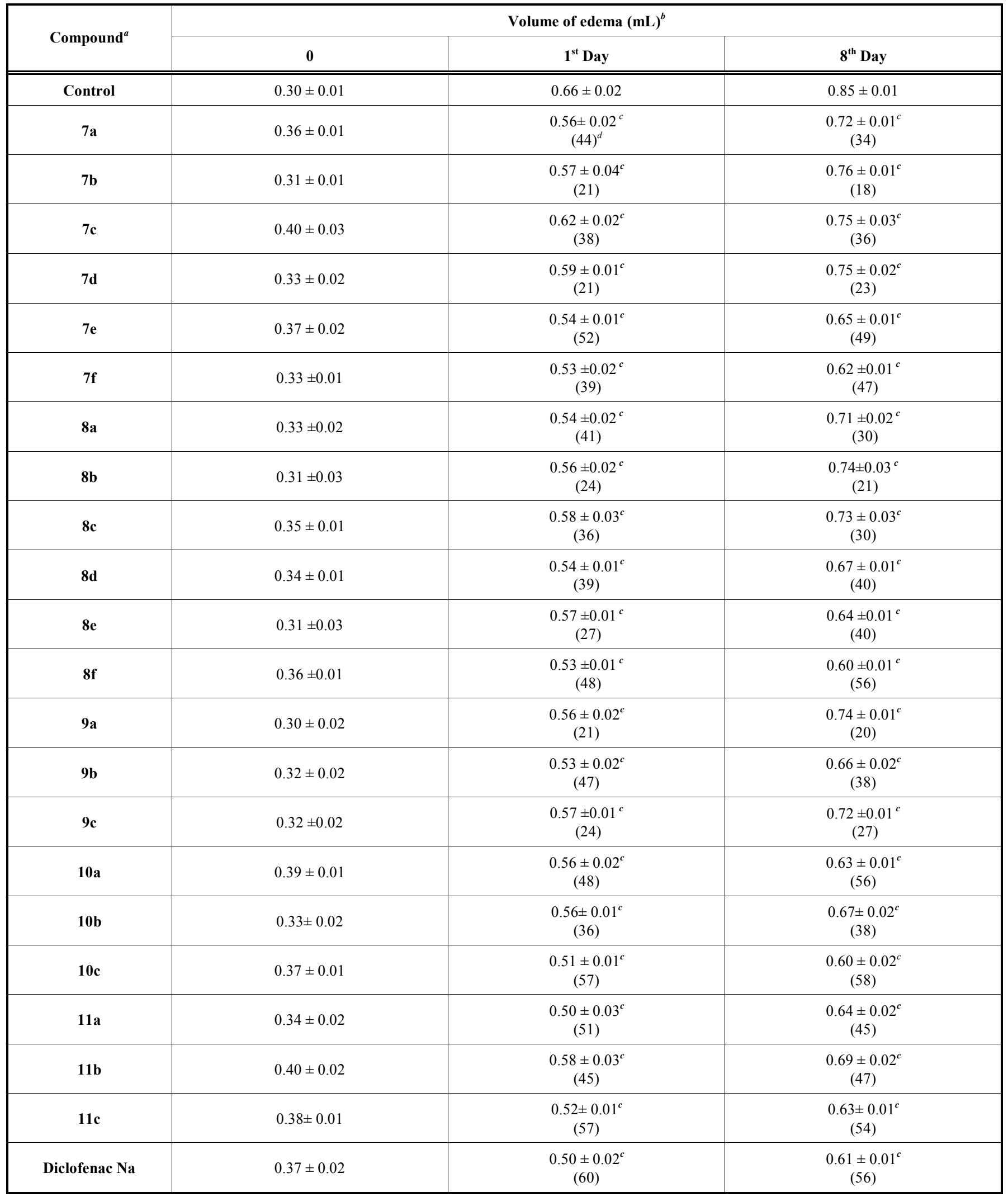

${ }^{\text {a }}$ Dose levels, po: test compounds (20 mg / kg b.wt.), diclofenac Na(20 mg / kg b.wt.).

${ }^{\mathrm{b}}$ Values are expressed as mean \pm S.E. (Number of animals $\mathrm{N}=5$ rats).

${ }^{\mathrm{c}}$ Significantly different compared to corresponding control.P $\leq 0.05$.

${ }^{\mathrm{d}}$ Between parentheses (Percentage anti-inflammatory activity, AI \%). 


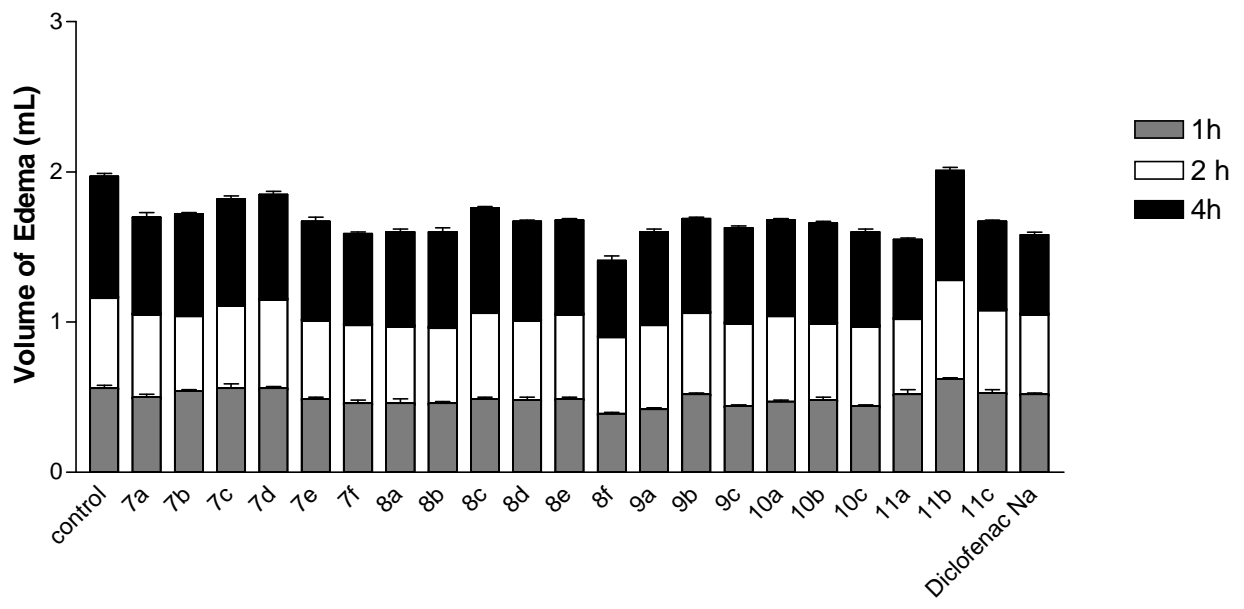

Test Compounds

Fig. (3). Anti-inflammatory activity(AI) of the newly synthesized compounds in formalin-induced rat paw edema bioassay (acute inflammatory model).

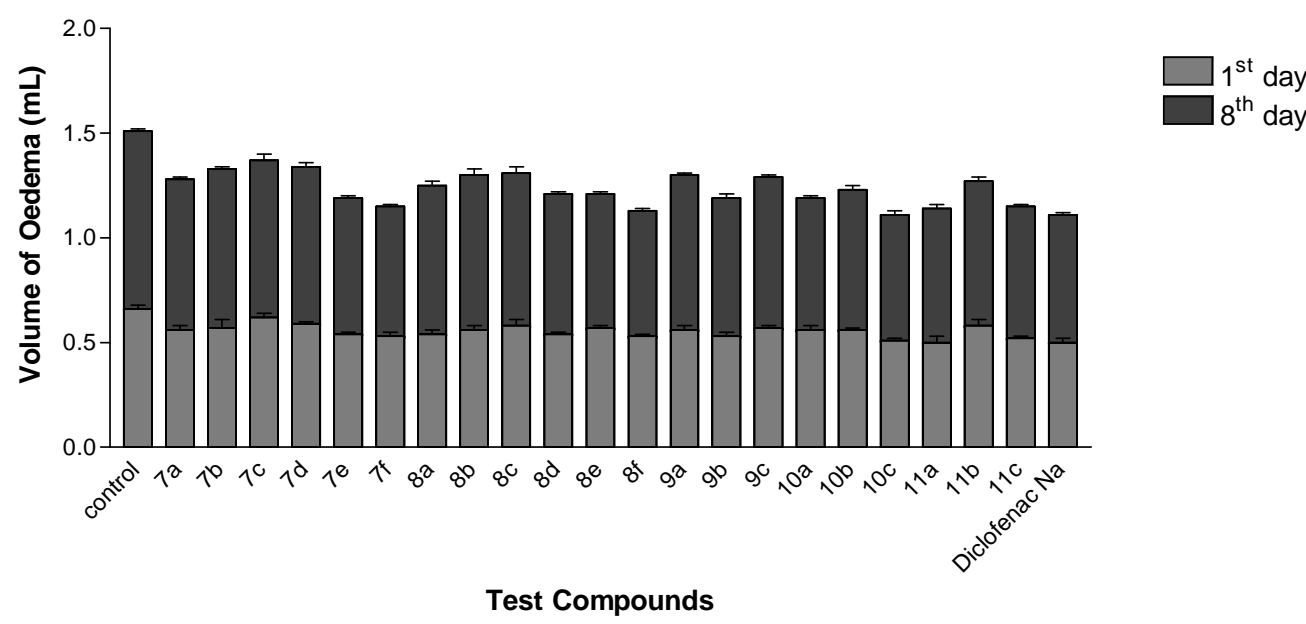

Fig. (4). Anti-inflammatory Activity of the newly synthesized compounds in formalin-induced rat paw edema bioassay (sub-acute inflammatory model).

would be less effective in controlling chronic inflammatory conditions.

\section{Ulcerogenic Activity}

The tested compounds namely; 7f, 8f, 10a, 10c, 11a and 11c that exhibited promising anti-inflammatory profiles in the pre-mentioned animal models were further evaluated for their ulcerogenic potential in rats [20]. Gross observation of the isolated rat stomach showed a normal stomach texture for compounds 10a ,10c and 11c with no observable hyperemia indicating a superior GI safety profile (no ulceration) in the population of the test animals at an oral dose of $300 \mathrm{mg} / \mathrm{kg}$, when administered twice at $2 \mathrm{~h}$ intervals in fasted rats. Whereas, compounds 7f, 8f, and 11a showed weak ulceration effect $(10 \%)$ as diclofenac sodium which was found to cause $10 \%$ ulceration under the same experimental conditions.

\section{Acute Toxicity}

The same selected compounds; 7f, 8f, 10a, 10c, 11a and 11c were further evaluated for their approximate acute lethal dose $\mathrm{ALD}_{50}$ in male rats using a literature method [21]. The results indicated that all of the tested compounds proved to be non-toxic and are well tolerated by the experimental animals. The compounds showed a high safety margin when screened at graded doses $(0.1-0.4 \mathrm{~g} / \mathrm{kg}$, po), where their $\mathrm{ALD}_{50}$ values were found to be $>0.4 \mathrm{~g} / \mathrm{kg}$.

\section{Analgesic Activity}

The analgesic activity of the synthesized compounds was evaluated using the rat tail withdrawal technique in response to immersion in water at $55{ }^{\circ} \mathrm{C}$ [22] using diclofenac $\mathrm{Na}$ as a reference drug $(20 \mathrm{mg} / \mathrm{kg}$, po). The analgesic activity was measured at $1-4 \mathrm{~h}$ time intervals after pain induction. The results were recorded as the average values of five administrations and the percentage increase of the reaction time in comparison with the basal values. The results were presented in (Table 3) and expressed as means $\pm \mathrm{SE}$.

A comparative study of the analgesic activity of the test compounds relative to the reference drug at different time intervals revealed the following: 
Table 3. Analgesic Activity of the Newly Synthesized Compounds Using the Rat Tail Withdrawal Technique

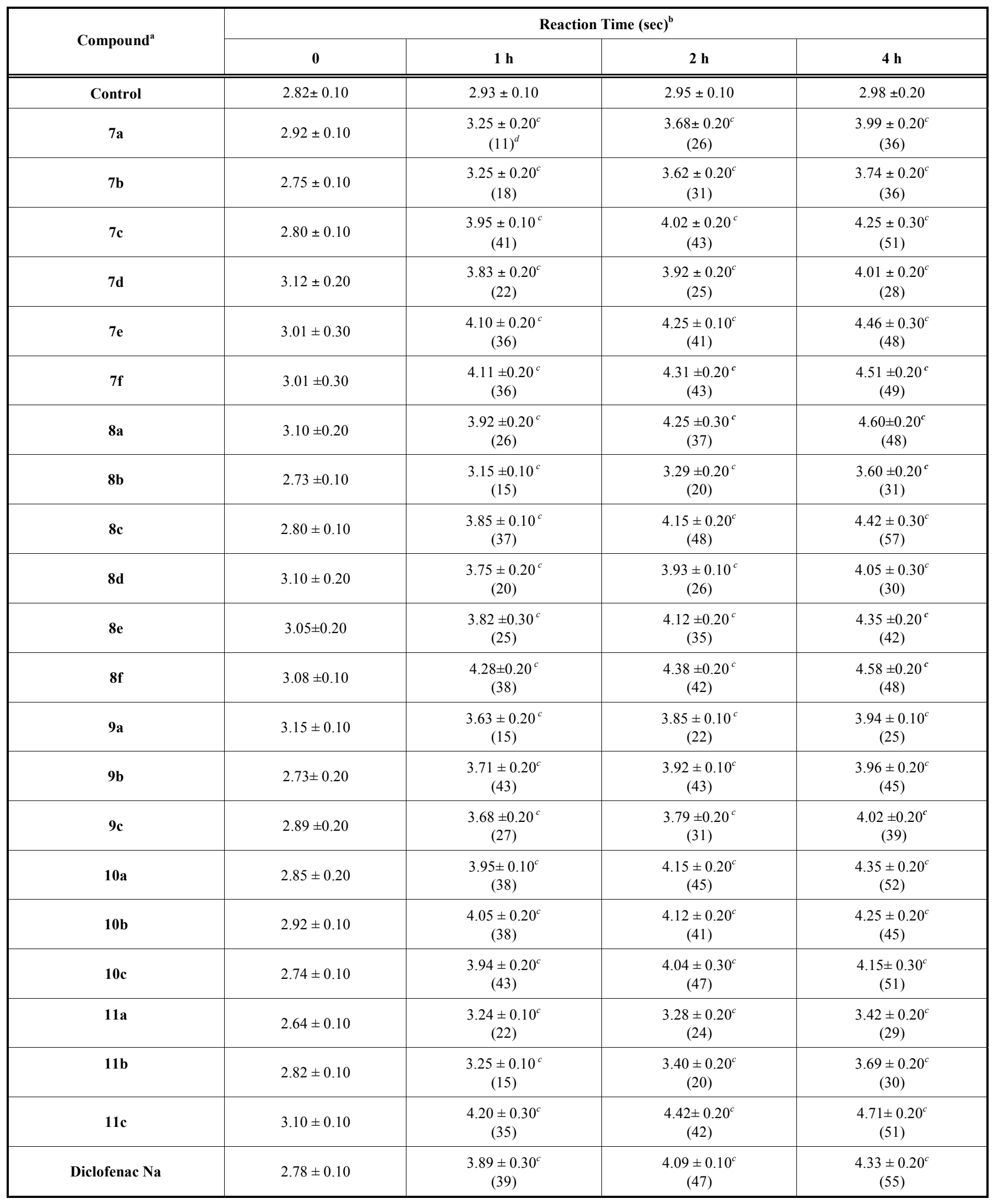

${ }^{\mathrm{a}}$ Dose levels, po: test compounds ( $20 \mathrm{mg} / \mathrm{kg}$ b.wt.), diclophenac Na (20 mg / kg b.wt.).

${ }^{\mathrm{b}}$ Values are expressed as mean \pm S.E. (Number of animals $\mathrm{N}=5$ rats).

${ }^{\mathrm{c}}$ Significantly different compared to corresponding control.P $\leq 0.05$.

${ }^{\mathrm{d}}$ Between parentheses (Percentage analgesic activity). 
After 1h, thienotriazolopyrimidines 7c, 9b and 10c exhibited potent analgesic activity (41, 43 and $43 \%$ respectively) compared to diclofenac $\mathrm{Na}(39 \%)$, while 7e, 7f, 8c, 8f, 10a, $10 b$ and 11c showed analgesic activity nearly equivalent (35-38\%) to diclofenac $\mathrm{Na}$.

After $2 \mathrm{~h}$, the results showed that thienotriazolopyrimidines 8c, 10a and 10c were found to be nearly equipotent (45$48 \%$ ) to the reference drug (47\%), whereas, 7c, 7e, 7f, 8 , 9b, 10b and 11c showed moderate activity (41-43\%).

After $4 \mathrm{~h}$, the thienotriazolopyrimidine 8c showed higher analgesic activity $(57 \%)$ compared to the reference drug $(55 \%)$, whereas $7 \mathbf{c}, 10 a, 10 c$ and 11c were found to be nearly equipotent or slightly less potent $(51-52 \%)$ than the reference drug. The rest of the tested compounds showed variable degree of analgesic activities ranging between weak to moderate (25-45\%). Results are illustrated by (Fig. 5).

Collectively, the thienotriazolopyrimidines derivatives 7c, 8c, 10a, 10c and 11c were proved to be the most potent analgesic agents with a delayed onset of action (percentage increase of reaction time (51-57\% after $4 \mathrm{~h}$ ) compared to diclofenac $\mathrm{Na}$ (percentage increase of reaction time $(55 \%$ after $4 \mathrm{~h}$ ).

\section{Docking Studies}

Molecular docking studies of compounds 10a, 10c and 11c were performed using Molecular Operating Environment (MOE-Dock 2008) module [23] in order to rationalize the obtained biological results. Molecular docking studies further helps in understanding the various interactions between the ligand and enzyme active site in detail. The determination of the three-dimensional co-crystal structure of COX-2 complex with Diclofenac Na (Fig. 6; PDB ID code: 1PXX) has led to the development of a model for the topography of the NSAIDs binding site in human COX-2 [26]. Therefore, herein we performed the docking studies using this COX-2 co-crystal structure with Diclofenac $\mathrm{Na}$ as a template. (Figs. 7-9) show the binding interactions of compounds 10a, 10c and $11 \mathrm{c}$ to the active site of COX-2, respectively, where they exhibited some similar interactions as with Diclofenac Na. Compound 10a displayed three hydrogen bond interactions with Arg 120 and Tyr 355 in addition to hydrophobic interactions with His 90, Arg 120, Val 349, Leu 352, Ser 353, Tyr 355, Phe 381, Leu 384, Tyr 385, Trp 387, Arg 513, Phe 518, Met 522, Val 523, Gly 526, Ala 527, Ser 530 and Leu 531. On the other hand, compound 10c showed four hydrogen bond interactions with Tyr 355 and Arg 120, in addition to hydrophobic interactions with His 90, Met 113, Arg 120, Ile 345, Val 349, Leu 352, Ser 353, Tyr 355, Leu 359, Phe 381, Leu 384, Tyr 385, Trp 387, Arg 513, Phe 518, Met 522, Val 523, Gly 526, Ala 527 and Leu 531. Compound 11c displayed two hydrogen bond interactions with Tyr 355 and Ser 530 , in addition to hydrophobic interactions with His 90, Leu 93, Tyr 115, Val 116, Arg 120, Val 349, Leu 352, Ser 353, Tyr 355, Leu 359, Leu 384, Tyr 385, Trp 387, Phe 518, Met 522, Val 523, Gly 526, Ala 527, Ser 530 and Leu 531.

\section{CONCLUSION}

The present study reports the synthesis and biological evaluation of 21 newly synthesized thienotriazolopyrimidines as anti-inflammatory and analgesic agents. The obtained results clearly revealed that most of the tested compounds showed better activity at the acute inflammatory model than the subacute one in addition to good analgesic profile. Moreover, the 8-\{[2-(phenylamino)-2-oxoethyl $]$ sulfonyl $\} \mathbf{8 a}$ and the 8-\{[2-(4-methoxyphenyl)ethylamino)-2-oxoethyl $]$ sulfonyl $\} \mathbf{8 f}$ derivative exhibited better anti-inflammatory activity at the acute model than its sulfanyl analogs $\mathbf{7 a}$ and $\mathbf{7 f}$ while the 8-methylsulfonyl $\mathbf{1 0 a}$ and 8-benzylsulfonyl 10c derivatives exhibited better anti-inflammatory activity at the acute model than its corresponding sulfanyl analoges 9a and 9c. Inaddition, replacement of the $\mathrm{S}$ methyl group at compound 9a with secondary amines enhances the antiinflammatory activity specially the N-phenylpiperazine derivative 11c. Collectively, thienotriazolopyrimidines 10a, 10c and 11c were proved to display distinctive antiinflammatory activity at both models as well as good analgesic profile with a delayed onset of action.

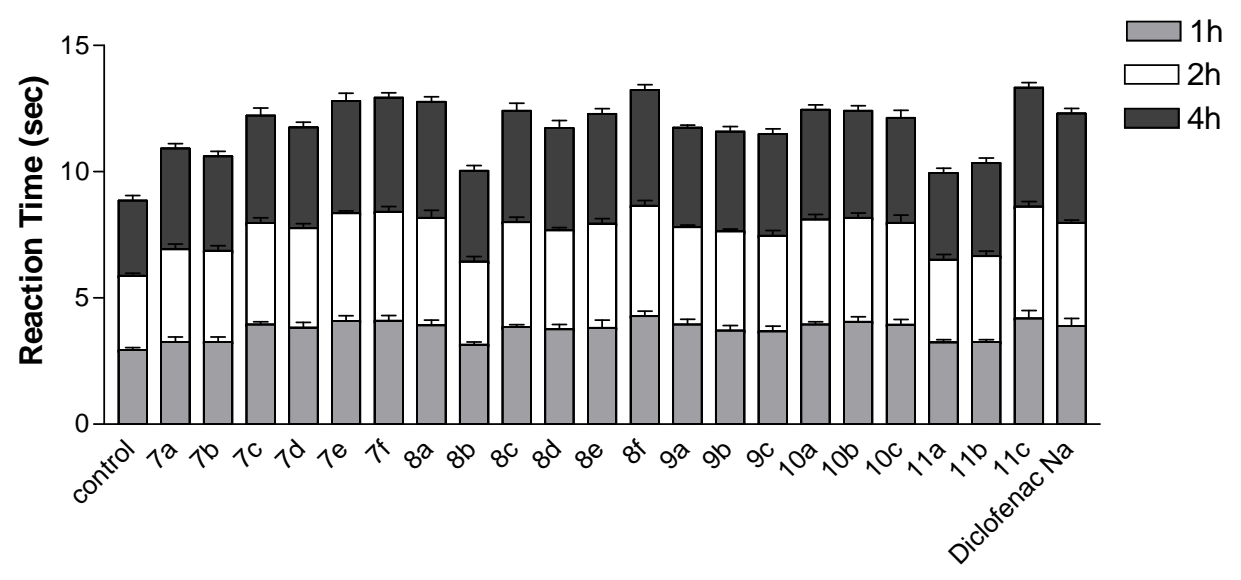

Test Compounds

Fig. (5). Analgesic activity of the newly synthesized compounds using the rat tail withdrawl technique. 

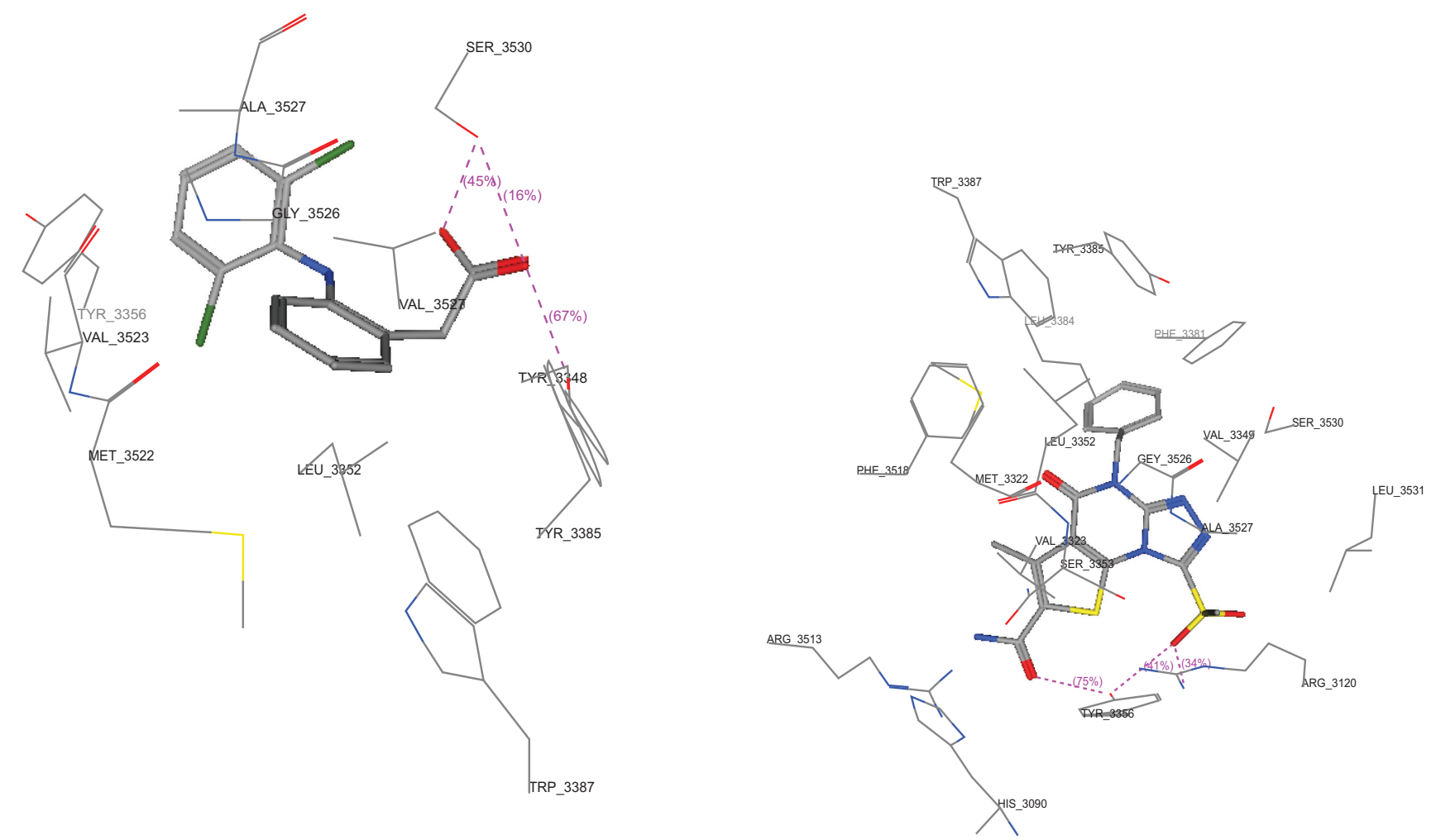

Fig. (6). 3D View from a molecular modeling study, of the minimum-energy structure of the complex of Diclofenac Na docked in COX-2 (PDB ID: 1PXX). Viewed using Molecular Operating Environment (MOE) module.

Fig. (7). 3D View from a molecular modeling study, of the minimum-energy structure of the complex of 10a docked in COX-2 (PDB ID: 1PXX). Viewed using Molecular Operating Environment (MOE) module.

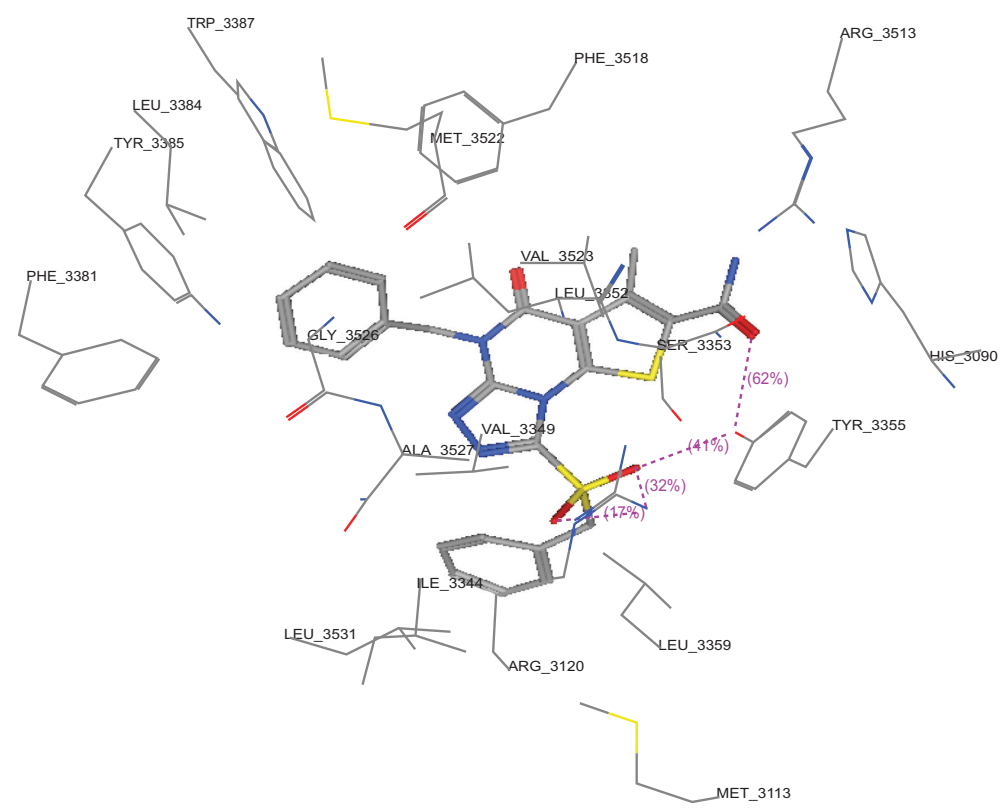

Fig. (8). 3D View from a molecular modeling study, of the minimum-energy structure of the complex of 10c docked in COX-2 (PDB ID: 1PXX). Viewed using Molecular Operating Environment (MOE) module. 


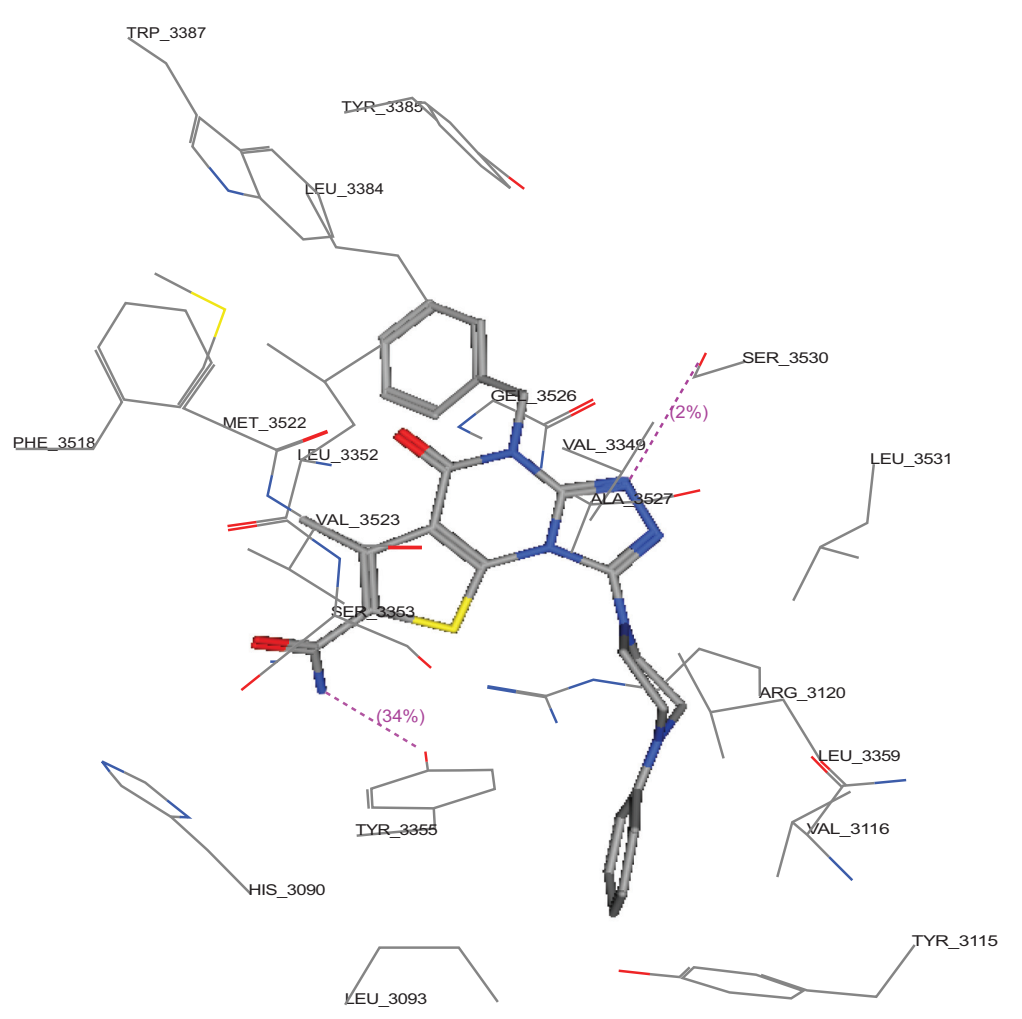

Fig. (9). 3D View from a molecular modeling study, of the minimum-energy structure of the complex of 11c docked in COX-2 (PDB ID: 1PXX). Viewed using Molecular Operating Environment (MOE) module.

Additionally, the active compounds revealed good GI safety profile and are well tolerated by experimental animals with high safety margin $\left(\mathrm{ALD}_{50}>0.4 \mathrm{~g} / \mathrm{kg}\right)$. Docking studies for compounds 10a, 10c and 11c with COX-2 enzyme (PDB ID code: 1PXX) showed good binding profile. Finally, these compounds represent new structure scaffolds that could be further optimized for future development of more potent anti-inflammatory and analgesic agents.

\section{CONFLICT OF INTEREST}

The authors declared no conflicts of interest. The authors alone are responsible for the content and writing of the paper.

\section{ACKNOWLEDGEMENTS}

Declared none.

\section{REFERENCES}

[1] Bombardier, C.; Laine, L.; Reicin, A.; Shapiro, D.; Burgos-Vargas, R.; Davis, B.; Day, R.; Bosi Ferraz, M.; Hawkey, C.J.; Hochberg, M.C.; Kvien, T.K.; Schnitzer, T.J. Comparison of upper gastrointestinal toxicity of rofecoxib and naproxen in patients with rheumatoid arthritis. N. Engl. J. Med., 2000, 343, 1520-1528. [2] Silverstein, F.E.; Faich, G.; Goldstein, J.L.; Simon, L.S.; Pincus,
T.; Whelton, A.; Makuch, R.; Eisen, G.; Agrawal, N.M.; Stenson, W.F.; Burr, A.M.; Zhao, W.W.; Kent, J.D.; Lefkowith, J.B.; Verburg, K.M.; Gies, G.S. Gastrointestinal toxicity with celecoxib vs nonsteroidal anti-inflammatory Drugs for osteoarthritis and rheumatoid arthritis: the class study. A randomized controlled trial. $J$. Am. Med. Assoc., 2000, 284, 1247-1255.

[3] Rashad, A.E.; Shamroukh, A.H.; Abdel-Megeid, R.E.; Mostafa, A.; El-Shesheny, R.; Kandeil, A.; Ali, M.A.; Banert, K. Synthesis and screening of some novel fused thiophene and thienopyrimidine derivatives for anti-avian influenza virus (H5N1) activity. Eur. $J$. Med. Chem., 2010, 45, 5251-5257.

[4] Rashad, A.E.; Ali, M.A. Synthesis and antiviral screening of some thieno[2,3-d] pyrimidine nucleosides. Nucleosides Nucleotides, 2006, $25,17-28$.

[5] Habib, N. S.; Soliman, R.; El-Tombary, A. A.; El-Hawash, S. A. Shaaban, O. G. Synthesis and biological evaluation of novel series of thieno[2,3-d]pyrimidine derivatives as anticancer and antimicrobial agents. Med. Chem. Res., 2013, 22, 3289-3308.

[6] Soliman, R.; Habib, N.S.; El-Tombary, A.A.; El- Hawash, S.A.; Shaaban, O.G. Synthesis of tetrahydrobenzothieno[2,3d]pyrimidine and tetrahydro-benzothieno[3,2-e]-[1,2,4]triazolo[4,3c] pyrimidine Derivative as potential antimicrobial agents. Sci. Pharm., 2009, 77, 755-773.

[7] Bhuiyan, M.D.; Rahman, K.M.; Hossain, M.D.; Rahim, A.; Hossain, M.I.; Abu Naser, M. Synthesis and antimicrobial evaluation of some new thienopyrimidine derivatives. Acta. Pharm., 2006, 56, 441-450.

[8] Hegab, M.I.; Hassan, N.A.; Rashad, A.E.; Fahmy, A.A.; AbdelMegeid, F.M.E. Synthesis, reactions, and antimicrobial activity of some fused thieno[2,3-d]pyrimidine derivatives. Phosphorus, Sulfur Silicon, 2007, 182, 1535-1556.

[9] Alqasoumi, S.I.; Ragab, F.A.; Alafeefy, A.M.; Galal, M.; Ghorab, M.M. Radioprotective and antitumor activity of some novel amino acids and imidazoles containing thieno[2,3-d]pyrimidine moiety. Phosphorus,Sulfur Silicon, 2009, 184, 3241-3257. 
[10] Munchhof, M. J.; Beebe, J. S.; Casavant, J. M.; Cooper, B. A.; Doty, J. L.; Higdon, R. C.; Hillerman, S. M.; Soderstrom, C. I.; Knauth, E. A.; Marx, M. A.; Rossi, A. M.; Sobolov, S. B.; Sun, J. Design and SAR of thienopyrimidine and thienopyridine inhibitors of VEGFR-2 kinase activity. Bioorg. Med. Chem. Lett., 2004, 14, 21-24.

[11] Sleebs, B. E.; Nikolakopoulos, G.; Street, I. P.; Falk, H.; Baell, J. B. Identification of 5,6-substituted 4-aminothieno[2,3-d]pyrimidines as LIMK1 inhibitors. Bioorg. Med. Chem. Lett., 2011, 21, 5992-5994.

[12] Alagarsamy, V.; Vijayakumar, S.; Raja Solomon, V. Synthesis of 2-mercapto-3-substituted-5,6-dimethylthieno[2,3-d]pyrimidin$4(3 \mathrm{H})$-ones as new analgesic, anti-inflammatory agents. Biomed. Pharmacother., 2007, 61, 285-291.

[13] El-Ansary, A.K.; Omar, A.H. Synthesis and anti-inflammatory activity of some thieno [2,3-d] pyrimidinone derivatives. Bull. Fac. Pharm. Cairo Univ. 2001, 39, 17-25.

[14] Alagarsamy, V.; Meena, S.; Ramseshu, K.V.; Solomon, V.R.; Thirumurugan, K.; Dhanabal, K.; Murugan, M. Synthesis, analgesic, anti-inflammatory, ulcerogenic index and antibacterial activities of novel 2-methylthio-3-substituted-5,6,7,8-tetrahydrobenzo (b) thieno[2,3-d]pyrimidin-4(3H)-ones. Eur. J. Med. Chem., 2006, 41, 1293-1300.

[15] Ashalatha, B.V.; Narayana, B.; Vijaya Raj, K.K.; Suchetha Kumari, N. Synthesis of some new bioactive 3-amino-2-mercapto5,6,7,8-tetrahydro[1] benzothieno [2,3-d] pyrimidin-4(3H)-one derivatives. Eur. J. Med. Chem., 2007, 42, 719-728.

[16] Modica, M.; Santagati, M.; Santagati, A.; Cutuli, V.; Mangano, N.; Caruso, A. Synthesis of new $[1,3,4]$ thiadiazolo[3,2-a]thieno[2,3d]pyrimidinone derivatives with antiinflammatory activity. Pharmazie, 2000, 55, 500-502.

[17] Rizk, O.H.; Shaaban, O.G.; El-Ashmawy, I.M. Design, synthesis and biological evaluation of some novel thienopyrimidines and fused thienopyrimidines as anti-inflammatory agents. Eur. J. Med. Chem., 2012, 55, 85-93.

[18] Ashour, H.M.A.; Shaaban, O.G.; Rizk, O. H.; El-Ashmawy, I. M. Synthesis and biological evaluation of thieno $\left[2^{\prime}, 3^{\prime}: 4,5\right]$ pyrimido[1,2-b][1,2,4]triazines and thieno[2,3-d][1,2,4] triazolo[1,5-a] pyrimidines as anti-inflammatory and analgesic agents. Eur. J. Med. Chem., 2013, 4, 341-351

[19] Hosseinzadeh, H.; Younesi, H.M. Antinociceptive and antiinflammatory effects of Crocus sativus L. stigma and petal extracts in mice. BMC Pharmacol., 2002, 2, 1-2.

[20] Daidone, G.; Maggio, B.; Raffa, D.; Plescia, S.; Bajardi, M.L.; Caruso, A.; Cutuli,V.M.C.; Amico-Roxas, M. Synthesis and pharmacological study of ethyl 1-methyl-5-[2-substituted-4-oxo-3(4H)quinazolinyl]-1H-pyrazole-4-acetates. Eur. J. Med. Chem., 1994, 29, 707-711.

[21] Verma, M.; Tripathi, M.; Saxena, A.K.; Shanker, K. Antiinflammatory activity of novel indole derivatives. Eur. J. Med. Chem., 1994, 29, 941-946.

[22] Jansem P.A.J.; Jagenav, A.H. A new series of potent analgesics: Dextro-2,2-diphenyl-3-methyl-4- morpholinobutyryl pyrrolidine and related amides. J. Pharm. Pharmacol., 1959, 9, 381.

[23] Molecular Operating Environment (MOE) version. Chemical Computing Group, Inc, Montréal, Canada, 2008 http://www.chemcomp.com

[24] Baraldi, P. G.; Preti, D.; Tabrizi, M. A.; Fruttarolo, F.; Saponaro, G.; Baraldi, S.; Romagnoli, R.; Moorman, A. R.; Gessi, S. Varani, K.; Borea P. A. N ${ }^{6}$-[(Hetero)aryl/(cyclo)alkyl-carbamoyl-methoxyphenyl]-(2-chloro)-5- $N$-ethylcarboxamido-adenosines: The first example of adenosine-related structures with potent agonist activity at the human $\mathrm{A}_{2 \mathrm{~B}}$ adenosine receptor. Bioorg. Med. Chem., 2007, 15, 2514-2527.

[25] El-Emam A. A.; Ibrahim, T.M. Synthesis and anti-inflammatory and analgesic activity of some 3-(1-adamantyl)-4-substituted-5mercapto-1,2,4-triazoles. Arzneim. Forsch., 1991, 41, 1260.

[26] Rowlinson, S.W.; Kiefer, J.R.; Prusakiewicz, J.J.; Pawlitz, J.L.; Kozak, K.R.; Kalgutkar, A.S.; Stallings, W.C.; Kurumbail, R.G.; Marnett, L.J. A novel mechanism of cyclooxygenase-2 inhibition involving interactions with Ser-530 and Tyr-385. J. Biol. Chem., 2003, 46, 45763-9.

(C) Shaaban et al.; Licensee Bentham Open.

This is an open access article licensed under the terms of the Creative Commons Attribution Non-Commercial License (http://creativecommons.org/licenses/by-nc/3.0/) which permits unrestricted, non-commercial use, distribution and reproduction in any medium, provided the work is properly cited. 